\title{
The role of crowding in parallel search: Peripheral pooling is not responsible for logarithmic efficiency in parallel search
}

\author{
Anna Madison ${ }^{1}$ - Alejandro Lleras ${ }^{1} \cdot$ Simona Buetti $^{1}$
}

Published online: 15 November 2017

(C) The Psychonomic Society, Inc. 2017

\begin{abstract}
Recent results from our laboratory showed that, in fixed-target parallel search tasks, reaction times increase in a logarithmic fashion with set size, and the slope of this logarithmic function is modulated by lure-target similarity. These results were interpreted as being consistent with a processing architecture where early vision (stage one) processes elements in the display in exhaustive fashion with unlimited capacity and with a limitation in resolution. Here, we evaluate the contribution of crowding to our recent logarithmic search slope findings, considering the possibility that peripheral pooling of features (as observed in crowding) may be responsible for logarithmic efficiency. Factors known to affect the strength of crowding were varied, specifically: item spacing and similarity. The results from three experiments converge on the same pattern of results: reaction times increased logarithmically with set size and were modulated by lure-target similarity even when crowding was minimized within displays through an inter-item spacing manipulation. Furthermore, we found logarithmic search efficiencies were overall improved in displays where crowding was minimized compared to displays where crowding was possible. The findings from these three experiments suggest logarithmic efficiency in efficient search is not the result peripheral pooling of features. That said, the presence of crowding does tend to reduce search efficiency, even in "pop-out" search situations.
\end{abstract}

Keywords Visual search · Crowding · Parallel search · Parallel processing $\cdot$ Pop-out

Anna Madison

amadiso2@illinois.edu

1 Department of Psychology, University of Illinois at Urbana-Champaign, 603 E. Daniel St, Champaign, IL 61820, USA

\section{Introduction}

The literature using visual search as a tool to study visual attention has, by and large, converged on the idea of visual processing occurring in two sequential stages (Duncan \& Humphreys, 1989; Hoffman, 1979; Treisman \& Gelade, 1980; Wolfe, 1994; although see single-stage proposals such as Verghese, 2001). The first stage unfolds in parallel, such that, at all locations, items are processed simultaneously. It is often assumed this processing unfolds with unlimited capacity. In contrast, the second stage unfolds in a more limitedcapacity fashion, such that only one or possibly a few items are processed at a time (Hoffman, 1979; Hulleman \& Olivers, 2015; Jonides, 1983; Neisser, 1964; Itti \& Koch, 2000; Treisman \& Gelade, 1980; Wolfe \& Gray 2007; Zelinsky, 2008).

Traditionally, feature search tasks have been employed to study the first stage of processing. Feature search, or pop-out search, refers to a visual search task, which requires searching for a target with one unique feature among non-target objects, which do not contain the unique feature (Treisman \& Gelade, 1980; Treisman \& Gormican, 1988; Treisman \& Souther, 1985). A general rule of thumb in the literature has been to deem search "efficient" or "fairly efficient" when the linear search slope of the RT by set size function is numerically smaller than $10 \mathrm{~ms} /$ item (e.g., Haslam, Porter \& Rothschild, 2001; Wolfe \& Horowitz, 2004). Often, search slopes of such small magnitude are interpreted as not being meaningfully different from zero, and, thus, it is often said that RTs in pop-out search are unaffected by set size (and unfold in parallel). The underlying assumption is that the target is so visually distinct from the non-target objects that attention is guided towards the target location because of strong bottom-up signals. Some theories argue that the target is the sole item in a hypothesized "feature map" (e.g., Treisman and Gelade, 1980; Wolfe, 1994), 
while other theories assume efficient guidance is achieved because the target produces the largest local contrast signal in the search scene (e.g., Itti \& Koch, $2000 \& 2001$ ). Note that, traditionally, feature search has been investigated with often extreme levels of target-distractor dissimilarity (e.g., red target among green distractors or horizontal target among vertical distractors), and manipulated set sizes often include no more than three levels. These methodological choices, as it turns out, do not allow for an accurate characterization of the set size by reaction time function (Buetti et al., 2016).

In a series of experiments, the authors studied efficient search by including different levels of target-distractor similarity and increased set size levels to six (e.g., 1, 2, 5, 10,20,32). The results indicated that when the target is fixed across trials search slopes increased logarithmically with set size in efficient search tasks, and, importantly, search efficiency was influenced by targetdistractor similarity. In Experiment 1A, subjects searched for a red triangle target amongst orange diamonds, yellow triangles or blue circles. The orange diamonds resembled the target in both shape and color and they produced the steepest logarithmic slope. The yellow triangles only resembled the target in shape (not color) and produced an intermediate search slope. The blue circles shared neither shape nor color with the target and they produced the smallest logarithmic slope. To demonstrate that the ordering of the log slopes indexed the target-lure similarity and not some intrinsic visual characteristic of the lures themselves, in Experiment 1B, a blue semi-circle was used as target while keeping the same distractor stimuli. This manipulation reversed the similarity relation between target and distractors. Now, the blue circles were the most similar to the target and the results showed they produced the steepest logarithmic slopes. The yellow triangles and orange diamonds were less similar to the target and they produced shallower logarithmic slopes.

More recently, Wang, Buetti and Lleras (2017) followed up on the findings of Buettietal. (2016) to demonstrate that (1) the same findings can be observed with real-world objects, and (2) that the logarithmic slope for a specific target-distractor stimulus pair can meaningfully predict the processing time of that distractor, across experiments, with new participants, and more importantly, even when the distractor type is presented in novel and more complex contexts (i.e., when the distractors are presented in heterogeneous displays, surrounded by other types of distractors).

Buetti et al., (2016) referred to stage one in the traditional twostage architecture as the Screening stage, and to stage two as the Scrutiny stage. During Screening, a binary decision is reached at each location indicating whether that item is likely to be the target or not. When items are sufficiently different from the target template, those items can be discarded in parallel from contention. However, due to spatial resolution limitations in peripheral vision resulting from differential processing characteristics across the retina as a function of eccentricity (see Strasburger, Rentschler, \& Juttner 2011 for a review of peripheral processing limitations in human vision), when items are sufficiently similar to the target, a confident decision about those items cannot be made in this early parallel stage, and a more precise scrutiny of the information at that location is required to determine whether the item is indeed the target. This second stage of processing unfolds with limited capacity in serial manner, inspecting one or a few neighboring items at a time, and it has a self-terminating stopping rule: the stage terminates upon identification of the target item. In contrast to the Screening stage, the Scrutiny stage is proposed to have unlimited resolution, which means that analysis is sufficiently detailed to distinguish the target from non-targets. Within the framework proposed by Buetti et al. (2016), stimuli can be categorized in two classes: lures and candidates. Lures are stimuli that are rejected as non-targets during Screening. Lures are sufficiently dissimilar from the target as to not require detailed scrutiny. Candidates are stimuli that are rejected as non-targets only after Scrutiny. Indeed, candidates are sufficiently similar to the target as to require focused attention or eye movements to determine their identity (see also Lleras, Buetti \& Mordkoff, 2013; Neider \& Zelinsky, 2008; Wolfe, Võ, Evans \& Greene, 2011). In sum, in terms of the two-stage model of visual search, lure stimuli are processed by stage one, but not stage two, whereas candidates are processed by both stage one and stage two.

Finally, Buetti et al. (2016) demonstrated through mathematical modeling inspired by the seminal work by Townsend and Ashby (1983) that the logarithmic search slopes were consistent with a parallel, unlimited capacity processing of the display with an exhaustive stopping rule ${ }^{1}$. Thus, this finding was taken as evidence that each item on the display meaningfully contributed to reaction time performance. In addition, computational simulations demonstrated that the magnitude of the logarithmic slope in our tasks corresponded to the amount of perceptual evidence needed to discard a lure item during stage one: as lure-target similarity increases, more evidence is required to reject lures of that type, and this will result in steeper logarithmic slopes. Therefore, the authors proposed that the logarithmic slopes can be taken as a quantitative index of lure-target similarity.

A well-known visual processing phenomenon that was overlooked in Buetti et al.'s (2016) experiments is crowding. Crowding is commonly recognized as the deleterious effect of near-by objects on object recognition when objects appear in the periphery, and it is thought to be a fundamental constraint on vision (Levi, 2008; Pelli, 2008; Pelli, Palomares \& Majaj, 2004; Pelli \& Tillman, 2008; Whitney \& Levi, 2011). The displays used in Buetti et al.'s experiments were not constructed to

\footnotetext{
${ }^{1}$ The term 'unlimited capacity' refers to the fact that the rate of processing of individual items is not dependent on the total number of items that must be processed. 'Parallel' refers to simultaneous processing of all the items in the display. Some items may finish processing before others due to the stochastic nature of processing. The term 'exhaustive' refers to the stopping rule of the process. An exhaustive stopping rule indicates that processing continues until all the items are completely processed (even after the target has been processed). In the Appendix of Buetti et al. (2016), we demonstrate why logarithmic functions are good approximations for this architecture.
} 
minimize crowding, and, particularly at large set sizes, crowding is likely to have occurred. That is to say, as more items were added to the search display, the probability two items were positioned next to each and could interact with each other increased. This would result in little to no crowding at lower set sizes (small RT cost) and a large probability of crowding at higher set sizes (larger RT cost). Therefore, a natural question that arises is whether the presence of peripheral crowding in the displays was directly responsible for the logarithmic nature of the RT functions reported by Buetti and colleagues.

Bouma's important work on crowding in letter recognition provided crucial insights into the extent of spatial interactions in the periphery, which led to the idea of critical spacing (Bouma, 1970): critical spacing refers to the minimum distance that must separate objects such that they no longer have a deleterious effect on identification performance. Critical spacing follows Bouma's Law (Bouma, 1970; Freeman \& Pelli, 2007; Levi, 2008; Pelli, 2008; Pelli \& Tillman, 2008, but see Rosen, Chakravarthi, \& Pelli, 2014). Bouma's Law states the critical spacing between two objects in the visual field increases with eccentricity, and, in general, is about half of the eccentricity of the target object (Bouma, 1970; Levi, 2008; Pelli, 2008; Pelli \& Tillman, 2008; Toet \& Levi, 1992). Critical spacing is typically measured by the center-to-center spacing between objects (although see Rosen, Chakravarthi \& Pelli, 2014). Toet and Levi (1992) demonstrated critical spacing is not the same across all directions and positions in the visual field and is elliptical in shape, with larger critical spacing $(0.5 \times$ eccentricity) found for items positioned in the radial direction around the target (i.e., along the line that connects fixation to the target location) compared to items positioned in the tangential direction $(0.1 \times$ eccentricity, i.e., perpendicular to the radial direction $)$. Most important to the current paper, crowding can thus be minimized by using Bouma's Law to construct displays with adequate spacing between objects such as to minimize the effects of crowding on visual processing (Pelli \& Tillman, 2008). Note too that the extent of spatially mediated interactions in crowding objects does not seem to be affected by target size (Tripathy \& Cavanagh, 2002).

A second factor known to impact crowding is flanker-target similarity (Andriessen \& Bouma, 1976; Ester, Ziber \& Serences, 2015; Kooi, Toet, Tripathy \& Levi, 1994; Nazir, 1992; Põder, 2007; Zahabi \& Arguin, 2014). The more similar a flanking item is to the target, the stronger its crowding effect is on target recognition, and the more dissimilar the flanking item is to the target, the larger is the release from crowding (e.g., Kooi et al., 1994; Põder, 1997, Scolari, Kohnen, Barton \& Awh, 2007). For instance, Nazir (1992) evaluated the effect of formand size-similarity of flanking objects on identification of a Landolt ring. Flanking objects could be an ' $\mathrm{O}$ ' (similar size and form), an 'E' (similar size different form), or a bar (different size and form). When the Landolt ring was flanked by an ' $\mathrm{O}$ ', performance deteriorated by $79 \%$ compared to $58 \%$ when the object was an 'E'. Kooi et al. (1994) found similar results when investigating similarity along low-level features, such as contrast polarity, shape and orientation, on identification of a $\mathrm{T}$ flanked by three other T's. Põder (2007) also found reduced crowding effects when color was used to differentiate flankers from target.

Although crowding is a much-studied visual phenomena, there is no agreed understanding regarding the mechanisms behind it (e.g., Pelli, 2008; Tripathy \& Cavanagh, 2002). Prominent accounts propose crowding may result because of limitations in the resolution of spatial attention ( $\mathrm{He}$, Cavanagh, Intiligator, 1996; Intriligator \& Cavanagh, 2001), because of "feature pooling" (i.e., the proposal that in peripheral vision, features inside "pooling regions" are combined rather than processed independently, e.g., Balas, Nakano \& Rosenholtz, 2009; Parkes, Lund, Angelucci Soloman \& Morgan, 2001; Pelli \& Tillman, 2008; Rosenholtz, Huang, Ehinger, 2012; Rosenholtz, Huang, Raj, Balas \& Illie, 2012), or by probabilistic substitution (i.e., swapping of flanker and target features, e.g., Ester, Klee, \& Awh, 2014; Ester et al., 2015).

More directly relevant to the current work, Rosenholtz and colleagues proposed that crowding plays a unique role in determining processing efficiency in visual search and that the loss of information in the periphery due to crowding can be directly related to the efficiency at which observers search through a scene (Balas et al., 2009; Rosenholtz et al., 2012a,b). In their framework, Rosenholtz and colleagues propose peripheral vision compresses the visual input, as a likely strategy to overcome limited perceptual capacity. This compression process leads to patches of the scene being represented in terms of summary statistics. The size of these patches increase with eccentricity. Thus, necessarily, crowding in peripheral vision leads to ambiguities between the original visual input and the represented visual information. In this account, target-distractor similarity effects do not directly determine search efficiency. Rather, the authors suggest, visual search performance is determined by the ease with which we can discriminate in the periphery patches of summary statistics containing target features from patches that do not (Rosenholtz et al., 2012a,b). As we will present below, it is possible then that RTs increase in logarithmic fashion in efficient search because of peripheral pooling of lure stimuli, and not because of the processing architecture proposed by Buetti et al. (2016).

\section{Current study}

In Buetti et al. (2016), items were presented in a grid-like arrangement (shown in Fig. 1), and, as such, crowding of the target item in the display was possible, particularly at large set sizes. We used simulations to evaluate the likelihood of the target being crowded in the displays of Buetti et al. (2016). 
Bouma's law was used to determine if another item in the display could crowd the target with Bouma's proportional constant equal to 0.5 . The probability of a target being crowded by another item was close to $0.40 \%$ when set size was $2,11.03 \%$ for a set size of $5,36.77 \%$ for a set size of $10,62.36 \%$ for, and $73.66 \%$ for a set size of 32 . Thus, the probability of a lure spatially interacting with the target was low for set sizes 2-10, but increased with set size. Similarly, as set size increased, the likelihood of lures interacting with one another also increased.

Buetti et al. (2016) interpreted the logarithmic increases in RT as the signature of an exhaustive, unlimited capacity, parallel process with a resolution limitation. The goal of the current investigation was to determine whether these logarithmic increases in RT were instead the result of crowding, or the processing limitations that give rise to crowding. For instance, one could imagine that, as more and more lures are presented in the display, the peripheral pooling proposed by Rosenholtz and colleagues could have the effect of reducing the impact of any one lure item on performance ${ }^{2}$. In other words, the marginal cost of adding an element to the display might not be constant, but, rather, it might decrease with set size. This follows because at small set sizes, every additional item added to the display is more likely to fall in an empty pooling region. As a result, processing this additional element should carry some cost associated with having to compute and extract information from one additional pooling region that was not previously contributing to performance. However, when we add an item to a display that already contains many items, chances are it will fall inside a pooling region that already contains (and is pooling) identical items. Thus, this additional item would not alter the summary statistics of that pooling region (or only minimally so). As a result, the marginal cost of processing every additional (identical) lure to the display should be a decreasing function with increasing set size. This would then result in monotonically increasing, but negatively accelerated functions, much like a logarithmic function.

This possibility can easily be tested: if displays were created in accordance to Bouma's Law such that crowding was minimized, would the stimuli used by Buetti et al. still produce logarithmic RT functions? Or would they revert to linear functions, as has been typically described in the visual search literature for the past several decades? To evaluate the role that crowding played in producing logarithmic search efficiency in Buetti et al.'s (2016) data, here we used the same stimuli and compared search performance across two

\footnotetext{
${ }^{2}$ To be clear, peripheral pooling models do not (as of now) include temporal processing parameters. What follows is an extrapolation of how we believe the pooling models would deal with processing additional items on a display, depending on whether additional items are added to a pool that is already processing items or one that is empty. We assume that peripheral pooling regions all process information in parallel with some time cost.
}

different display arrangements: the original rectangular grid-like arrangement used in that study where inter-item crowding was possible (see Fig. 1a) and compared to a new display arrangement where inter-item spacing was sufficient to minimize crowding in the search displays (see Fig. 1b). If crowding is responsible for the logarithmic patterns observed in efficient search, then once crowding is minimized, RT performance should revert to being linear. However, if the logarithmic search slope is not the direct result of inter-item crowding in those displays, then we should once again find RTs increasing logarithmically with set size, in spite of the absence of crowding in the displays.

We conduced three experiments. In all conditions, participants were asked to find a red triangle and report the target's orientation. Two important design elements were included from Buetti et al. (2016): (1) inclusion of multiple set sizes which spanned a large range (1-32 items) to be able to meaningfully compare logarithmic and linear fits to the observed RT patterns, and (2) a manipulation of lure-target similarity to replicate the finding that lure-target similarity modulates the slope of the logarithmic curve. One-half of the trials items were presented in the rectangular grid where crowding was possible (henceforth referred to as "crowding-possible" display) and the other half of trials items were presented following a concentric arrangement that minimizes crowding in the display (henceforth referred to as "crowding-minimized" display). Importantly, within each experiment all conditions (lure type, set size, and display type) were randomly intermixed. RT was used as the main dependent variable of interest ${ }^{3}$.

\section{Experiment 1}

\section{Methods}

\section{Subjects}

All subjects completed the experiment in exchange for course credit in a Psychology class. The effect of display arrangement was a priori unknown, so a target sample size was chosen based on a similar power analysis performed for Experiment 1a in Buetti et al. (2016). That power analysis provided a target sample size for detecting the smallest effect of reaction time performance in the most dissimilar lures (blue circles). It revealed that with $80 \%$ power, 18 participants would be required to find an effect of lure type on search efficiency. Thus, we chose to keep the sample size

\footnotetext{
${ }^{3}$ Traditionally, measures such as accuracy and contrast threshold have been used to evaluate the effect of crowding on performance, though Zahabi and Arguin (2014) demonstrated that RT can also be used as a dependent measure when studying crowding.
} 


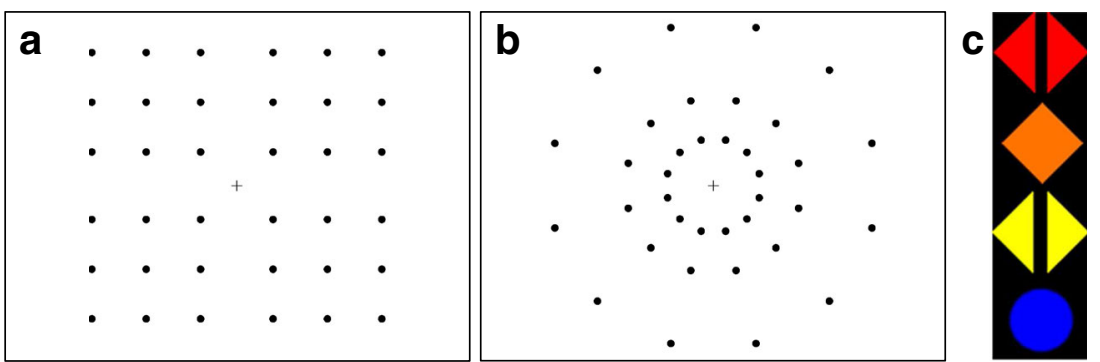

Fig. 1 a All possible item locations in a rectangular grid arrangement in which crowding was possible. b All possible item locations in a concentric grid arrangement, in which crowding was minimized. c Sample stimuli used across Experiments 1-3

used in Buetti et al. (2016) and collect data from 20 subjects. Data from the first 20 subjects meeting the inclusion criteria of Buetti et al. (2016) were analyzed: subjects were replaced for overall performance below $90 \%$ accuracy or who had mean RTs higher than 2 times the standard deviation of the mean RT for the group. This resulted in two subjects being replaced: one subject due to high error rate $(>10 \%)$. All subjects were tested for color blindness using Ishihara color plates.

\section{Apparatus and stimuli}

This experiment was run on a PC, using Matlab, and programmed with the Psychophysics Toolbox, version 3 (Brainard, 1997; Pelli, 1997). Subjects were seated $49 \mathrm{~cm}$ from a 20-inch CRT monitor (20 degrees of visual angle) in a dimly lit room. On every display, all items subtended .833 degrees of visual angle. Subjects were asked to locate the target, a red triangle pointing to the left or right, and to report the orientation. A target was presented on the display on every trial. Subjects were asked to respond with the right arrow key when the target was pointed to the right and left arrow key when the target was pointed to the left. Subjects used the pointer and middle fingers of their right hand to respond.

The lures could appear in two possible display arrangements: crowding-possible or crowding-minimized. (1) The crowding-possible display presented all items on an invisible $6 \times 6$ rectangular grid array (36 locations) with random jitter added, the same configuration used in Buetti et al. (2016). (2) The crowding-minimized display presented all the items on three concentric circles and was adapted from Santhi and Reeves (2004) with random jitter added. The circles were spaced approximately at 4.3, 8.3 and 15.6 degrees of visual angle, and each contain 12 possible locations, evenly spaced, 30 degrees apart (for a total of 36 locations across the three eccentricities). Critically, in the crowding-minimized display, the spacing between any two possible location points was greater than the boundary of crowding, as stated by Bouma's Law and increased with eccentricity (Pelli, 2008). Thus, this display was designed to minimize crowding within the display. Lure items could be either the orange diamonds or yellow triangles used in Buetti et al. (2016).

\section{Procedure}

Every trial began with a presentation of a fixation cross for $1 \mathrm{~s}$ followed by a search display, which remained on the screen until a response was given or until $5 \mathrm{~s}$ had past. There was an inter-trial interval of $1.5 \mathrm{~s}$. Displays could contain the target with 0 (target only condition), 1, 4, 9, 19, or 31 lure objects. Two levels of lure-target similarity were used: high similarity (for orange diamonds) and medium similarity (for yellow triangles). Error trials were followed by a short beep. Subjects' eye-movements were not monitored during trials and subjects were free to move their eyes throughout the trial.

\section{Design}

The display type (crowding-possible vs. crowding-minimized), lure set size $(0,1,4,9,19,31)$ and lure type (orange diamonds vs. yellow triangles) were manipulated within-subjects. Trial conditions were randomized for each subject and subjects were allowed to take a break every 77 trials. Participants completed 22 target-only trials for each display type (a total of 44 total target-only trials), and 44 trials each of the other conditions, for a total of 924 experimental trials. The first four participants completed 1050 trials of randomized conditions. Due to time constraints, we had to reduce the number of trials per session to 924 . As a result and for consistency, only the first 924 trials were analyzed from the first four participants to have comparable numbers of observations per participant.

\section{Results}

We first compared the fit of the linear and logarithmic bestfitting lines of the RT by set size function. Error rates were evaluated to rule out any speed-accuracy trade-offs. Next, we evaluated the effect of the display and lure-target similarity manipulations on the logarithmic search slopes with a repeated measure ANOVA. This was followed with planned comparisons using paired $t$ test between the logarithmic slopes for each display and lure type to evaluate lure-target similarity effects as well as effects of the display manipulation. Finally, 
we conducted an exploratory analysis to evaluate the effect of eccentricity on target processing times (Carrasco, Evert, Chang \& Katz, 1995; Carrasco, McLean, Katz \& Frieder, 1998; Carrasco \& Yeshurun, 1998). Violations of sphericity were corrected by adjusting the degrees of freedom following the Greenhouse-Geisser procedure. The effect sizes for the ANOVA analyses and the effect sizes for the $t$ test analyses are provided as Cohen's $d$.

\section{Search slopes}

First, we evaluated the search slope functions produced by each lure type in each display arrangement. Search functions included the target only datum, as we have previously demonstrated evidence of the logarithmic search functions converging on the target only conditions (Buetti et al., 2016). In Experiment 1, the search slopes produced by both display types were best approximated by logarithmic search functions. This is seen as higher $R$ squares for the logarithmic regression fit than the corresponding linear regression fit for all conditions (Table 1, Fig. 2). Replicating Buetti et al. (2016), the crowdingpossible displays with orange diamonds had a higher $R^{2}$ for the logarithmic fit $\left(R^{2}=0.9751\right)$ than the linear fit $\left(R^{2}\right.$ $=0.8224)$. This was also found for the crowding-possible displays with yellow triangles, which had a higher $R^{2}$ for the logarithmic fit $\left(R^{2}=0.7841\right)$ than the linear fit $\left(R^{2}=\right.$ 0.4812 ). New to this study, the crowding-minimized displays containing orange diamonds also had a higher $R^{2}$ for the logarithmic fit $\left(R^{2}=0.9753\right)$, than for the linear fit $\left(R^{2}=0.7976\right)$. The same results were found with yellow triangles: the $R^{2}$ for the logarithmic fit was larger $\left(R^{2}=\right.$ $0.9737)$ than the linear fit $\left(R^{2}=0.7687\right)$. The observed linear slopes for all the conditions were small and would be classified as reflecting "efficient" search using the 10 ms/item rule of thumb (e.g., Wolfe \& Horowitz, 2004), see Table 1.

\section{Logarithmic search slopes}

Next, we analyzed the individual logarithmic search slopes (shown in Fig. 2a): logarithmic functions including the target only datum were fit to each participant's data. The effect of crowding on search efficiency was evaluated by comparing the log slopes between the two display types for each lure type. A $2 \times 2$ repeated measure ANOVA was conducted to compare the effect of display type and lure type on the log slopes. Display type was found to have a significant impact on the logarithmic slopes, with crowding-minimized displays producing more efficient processing (i.e., smaller log slopes) than crowding-possible displays $\mathrm{F}(1,19)=15.23, P<$ 0.001 , Cohen's $d=1.79$. Lure type also had a significant effect on log slopes: lures with lower similarity to the target (blue circles) produced more efficient processing than lures with higher similarity to the target (orange diamonds) $\mathrm{F}(1,19)=12.75, P=0.0023$, Cohen's $\mathrm{d}=1.64$. The interaction between display type and lure type was not significant $\mathrm{F}(1,19)=1.68, P=0.081$, Cohen's $\mathrm{d}=0.59$.

The effect of crowding on processing efficiency was evaluated by comparing the logarithmic search slopes for each lure type between each display type (Fig. 3). A paired $t$ test revealed that orange diamond lures were processed more efficiently on crowding-minimized displays ( $31.3 \mathrm{~ms}$ per log unit) than on crowding-possible displays (46.7 ms per log unit), $\mathrm{t}(19)=3.52, P=0.0023$, Cohen's $\mathrm{d}=1.11$. Although there was a numerical difference between the processing efficiency for yellow triangles on crowding-minimized displays (19.5 ms per log unit) and on crowding-possible displays (26.4 ms per $\log$ unit), the difference was not significant, $\mathrm{t}(19)=1.6, P=$ 0.13 , Cohen's d $=0.51$.

The effect of lure-target similarity on processing efficiency was evaluated by comparing the logarithmic slopes within each display type. Lure-target similarity modulated the lure slopes for both display types. For the crowding-possible display, orange diamonds ( $46.7 \mathrm{~ms}$ per log unit) were processed less efficiently than the yellow triangles lures (26.4 ms per log

Table 1 Linear (in ms/item) and logarithmic search slopes (in ms/log unit) from Experiment 1 are shown for each lure and display types with the corresponding $R^{2}$

\begin{tabular}{|c|c|c|c|c|c|}
\hline & & Linear search slopes & $R^{2}$ & Log search slopes & $R^{2}$ \\
\hline \multirow[t]{2}{*}{ Orange lures } & Crowding-possible display & 4.72 & 0.8224 & 46.69 & 0.9751 \\
\hline & Crowding-minimized display & 3.11 & 0.7976 & 31.25 & 0.9753 \\
\hline \multirow[t]{2}{*}{ Yellow lures } & Crowding-possible display & 2.28 & 0.4812 & 26.41 & 0.7841 \\
\hline & Crowding-minimized display & 1.91 & 0.7687 & 19.47 & 0.9737 \\
\hline
\end{tabular}

The linear and log slopes are computed including the target only conditions 

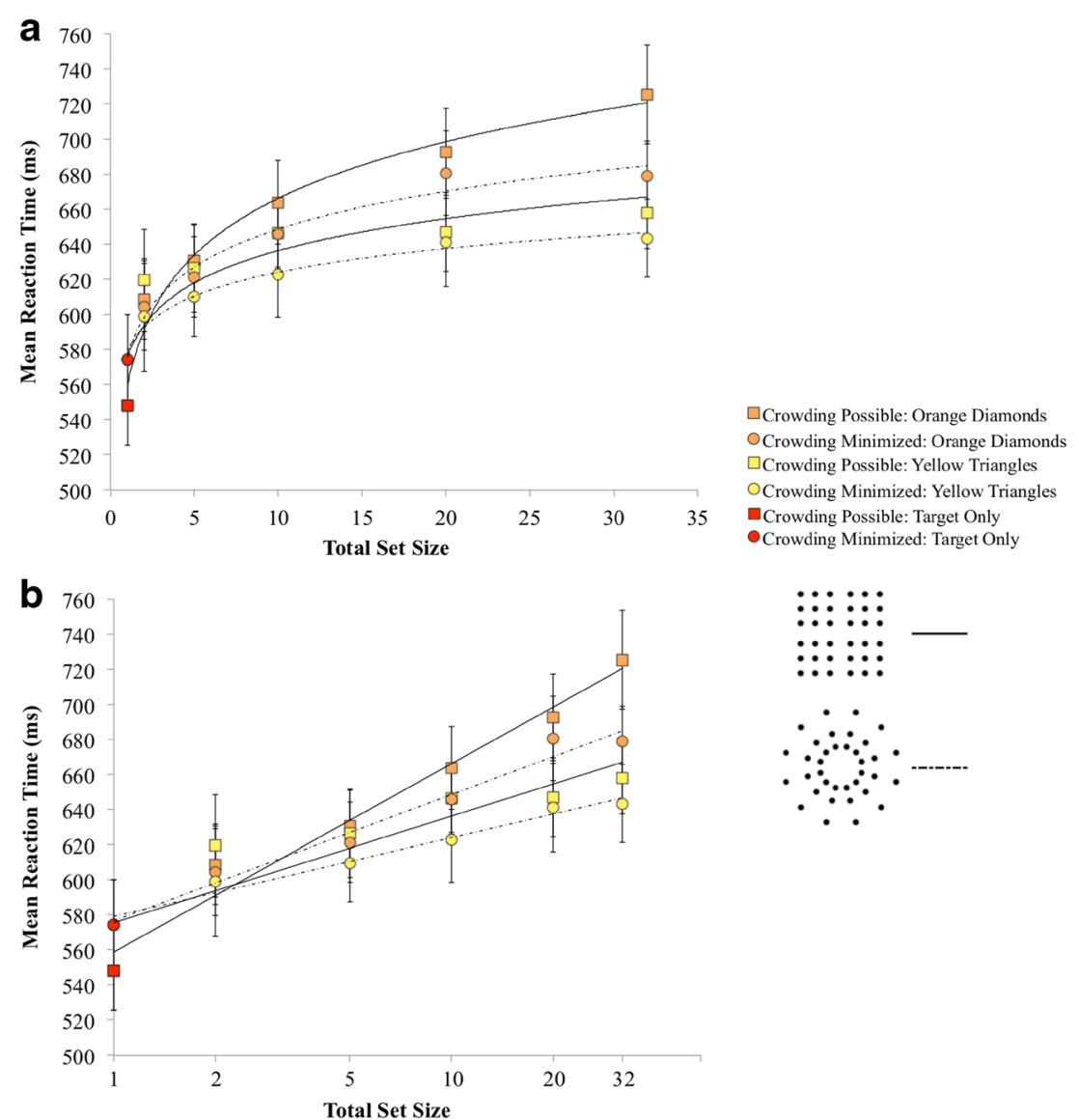

$\because:: \geq::$ $\because:: \because::$

$\cdot$

$\because \therefore 0^{\circ}$

$\therefore \therefore$.

$\because \because \because$ Fig. 2 a,b Search performance as a function of display type, set size and
lure type (lure-target similarity) from Experiment 1. Error bars Standard of items on the display and reaction time (RT; ms). b The same data plotted on logarithmic scale for set size with the corresponding total set error of the mean (SEM). a Search performance plotted on a linear scale for set size, showing a logarithmic relationship between the total number

unit), $\mathrm{t}(19)=3.03, P<0.001$, Cohen's $\mathrm{d}=0.96$. This was also true for the crowding-minimized display; orange diamonds (31.3 ms per log unit) were processed less efficiently than yellow triangles (19.4 ms per $\log$ unit), $\mathrm{t}(19)=2.85, P=$ 0.010 , Cohen's $d=0.90$.

\section{Error rates}

To rule out the possibility of a speed-accuracy trade-offs, error rates were also analyzed. Error rates were in general low (M: $2.21 \%$, SD: $0.52 \%$ ). A repeated measures ANOVA was conducted on the error rates with display (crowding possible or minimized), lure (orange diamonds or yellow triangles), and set size $(2,5,10,20,32)$ as within subject factors. Display $\mathrm{F}(1,19)=$ $0.086, P=0.77$, Cohen's d $=0.14$, lure $\mathrm{F}(1,19)=0.82, P=0.38$, Cohen's $\mathrm{D}=0.41$, and set size $\mathrm{F}(2.273,43.178)=0.11, P=0.91$, Cohen's $\mathrm{D}=0.16$ did not have a significant effect on error rate. All of the interactions were not significant $\mathrm{Fs}<2.5$ and $P_{\mathrm{S}}>0.07$. This suggests it is unlikely that a speed-accuracy trade-off was responsible for the non-linearities in the RT data. size conditions as labels

\section{Eccentricity effects}

Increases in RT performance with increases in target eccentricity have been previously reported (Carrasco, Evert, Chang \& Katz, 1995; Carrasco, McLean, Katz, Svetlana \& Frieder, 1998; Carrasco \& Yeshurun, 1998). Although not central to our study, in an exploratory analysis, the effect of target eccentricity on response times as a function of display type was evaluated. We collapsed across set size, lure type and position in the display to compute the average RT at each location in the display. This produced 12 observations per target position per subject. Each display arrangement was analyzed separately and the statistical testing for eccentricity effects was done within each display arrangement (Appendix 1: Fig. 8 and Fig. 9). The RTs at three different eccentricities from fixation were averaged within the crowding-possible display arrangement: the innermost four locations around fixation (7.6 degrees; near eccentricity), the outermost four locations near the outer corner of the display (20.1 degrees; far eccentricity), and the 


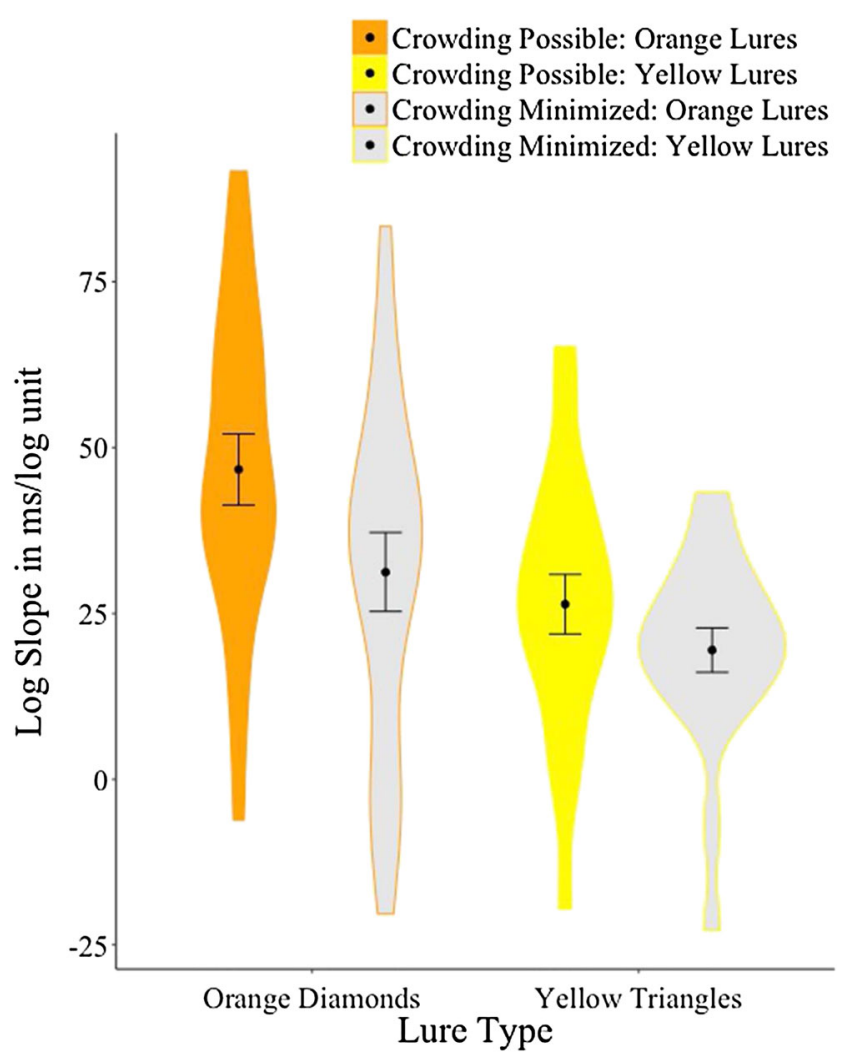

Fig. 3 Violin plots of the logarithmic slope coefficients from Experiment 1 are shown for the both display types grouped by lure type. Violin plots are similar to a box plot but have a kernel density function plotted vertically (and symmetrically) to indicate the probability density of the underlying data (i.e., individual subject means). The mean log slope is indicated by the dot on each plot. Error bars SEM

average of the four locations that fell in between the innermost and outermost locations along the diagonal that is created (14.0 degrees; middle eccentricity). The corresponding locations in the crowding-minimized display had three levels of eccentricity (4.3,8.3 and 15.6 degrees). Not surprisingly, eccentricity had a significant effect on RT in both the crowding-possible display, $\mathrm{F}(2,38)=$ 71.34, $P<0.001$, Cohen's $\mathrm{d}=0.98$, and the crowdingminimized display, $\mathrm{F}(2,38)=15.74, P<0.001$, Cohen's $\mathrm{d}=0.4935$.

\section{Discussion}

The results from Experiment 1 indicate the possibility that crowding on search displays can contribute to RT performance in parallel search tasks. Results showed crowding can modulate the efficiency of search when lure-target similarity is relatively high. However, crowding itself does not appear to be the cause of the logarithmic shape of RT functions in parallel search: even when crowding was minimized, RT functions remained logarithmic. As previously proposed (Buetti et al., 2016), the logarithmic search slope functions are most likely a result of the underlying unlimited parallel exhaustive processing from the first stage of visual selection.

Buetti et al. (2016) proposed that the logarithmic search slopes provide meaningful information about the extent of processing of different items, with higher logarithmic search slopes indicating a larger amount of evidence is required to classify a lure as non-target than when $\log$ slopes are small, corresponding to lures that require less evidence to be classified as non-target. The effect of display type on the logarithmic search slope for orange lures indicates search efficiency can be improved in crowding-minimized displays. That is, in crowdingpossible displays, subjects had to process items for longer amounts of time (i.e., gather more evidence) than in crowding-minimized displays. It is worth noting that in this experiment, all conditions were randomly intermixed so subjects could not anticipate which type of display they would see next. Overall, one may consider the log slopes measured in the crowding-minimized displays as perhaps a more direct estimate of the processing efficiency of a given lure type, free from any processing costs associated with crowding. Finally, the current results also replicated previous findings from our lab demonstrating the logarithmic search slopes in fixed-target efficient search are modulated by the lure-target similarity.

Logarithmic search slopes have been found by Carrasco and colleagues before, and have been largely attributed to eccentricity effects and limitation of spatial resolution (Carrasco et al., 1995, 1998; Carrasco \& Yeshurun, 1998). The eccentricity effects found in both display types indicate that eccentricity plays a role in the processing times of the target, even when crowding has been minimized. These results are consistent with previous findings of eccentricity affecting RT, and suggest even when crowding is minimized, eccentricity continues to influence RTs. Furthermore, these results are consistent with previous eccentricity findings where increasing critical spacing did not eliminate eccentricity effects, whereas scaling the size of the stimuli according to the cortical magnification factor did (Carrasco \& Frieder, 1997). The effect of eccentricity measured here was exploratory, as this experiment was not designed (or powered) to finely investigate eccentricity effects. That said, the presence of an eccentricity effect in both display conditions is indirect evidence that eye movements did not have a large contribution to search performance, and that participants were able to gather sufficient amounts of information before moving their eyes to the target. 


\section{Experiment 2}

Experiment 2 was conducted as a follow-up to Experiment 1, to both confirm and extend those findings to another lure type (blue circles) from our previous RT findings (Buetti et al., 2016). Thus, this experiment investigated whether the logarithmic nature of the RT functions is still preserved when crowding is minimized and lure-target similarity is extremely low. Lure-target similarity was manipulated in Experiment 2 by using yellow triangles (similar in shape, but not color) and blue circles (dissimilar in both shape and color). As in Experiment 1, subjects were asked to search for a red target to report its orientation. Everything else was identical to Experiment 1. We expected crowding to have a smaller impact on RTs and search efficiency since previous studies have found when objects are very dissimilar from the target crowding effects are reduced (Kooi et al., 1994; Põder, 1997; Zahabi \& Arguin, 2014).

\section{Methods}

Methods were identical to Experiment 1 except where noted.

\section{Subjects}

Data from a new set of 20 subjects from the same pool as Experiment 1 that met the same performance criterion were used for analysis. Two subjects were replaced for having overly long RTs (RTs $>$ than 2 SD above the mean RT of the group).

\section{Stimuli}

The orange lures used in Experiment 1 were replaced by blue circle lures.

\section{Design}

Participants completed 22 target-only trials for each display arrangement (a total of 44 total target-only trials), and 44 trials each of the other conditions, for a total of 924 experimental trials.

\section{Results}

\section{Search slopes}

A similar set of analyses as for Experiment 1 was conducted. Consistent with Experiment 1, the search slope functions for both the crowding-possible and crowding-minimized displays for both lure types were best approximated by logarithmic search functions (Table 2 and Fig. 4). This is seen as higher $R$-squares for the logarithmic search slopes functions compared to the linear search slope functions for all the tested conditions and replicating the results of Experiment 1. Most importantly, in the crowdingminimized displays with yellow triangles, the $R^{2}$ for the logarithmic fit was $R^{2}=0.9696$, which was higher than the linear fit $\left(R^{2}=\right.$ 0.7359). And, in the crowding-minimized displays with blue circles, the $R^{2}$ for the logarithmic fit was $R^{2}=0.8754$, which was higher compared to the linear fit $\left(R^{2}=0.6429\right)$. As in Experiment 1, the observed linear search slopes were small, less than $2.5 \mathrm{~ms} /$ item, for both lure types (shown in Table 2). Replicating our previous findings, this search task would be considered as efficient, according to the traditional rule-of-thumb definition of efficient search (Treisman \& Gelade, 1980; Treisman \& Sato, 1990; Wolfe, 1998).

\section{Logarithmic search slopes}

Next, we analyzed the individual logarithmic search slopes (shown in Fig. 4a) to evaluate the effect of crowding and lure-target similarity on processing efficiency. A $2 \times 2$ repeated measure ANOVA with display type and lure type as withinsubject factors was conducted on the log slopes. No effect of display type was found. Crowding-minimized displays produced similar processing efficiencies as crowding-possible displays $\mathrm{F}(1,19)=0.27, P=0.61$, Cohen's $\mathrm{d}=0.24$. But, an effect of lure type was found. Lures with lower similarity to the target (blue circles) produced more efficient processing

Table 2 Linear (in $\mathrm{ms} /$ item) and logarithmic search slopes (in $\mathrm{ms} / \log$ unit) from Experiment 2 are shown for each lure and display arrangements with the corresponding $R^{2}$

\begin{tabular}{llllll}
\hline & & Linear search slopes & $R^{2}$ & Log search slopes & $R^{2}$ \\
\hline Yellow lures & Crowding-possible display & 2.38 & 0.6896 & 25.0 & 0.9225 \\
& Crowding-minimized display & 2.46 & 0.7359 & 25.66 & 0.9696 \\
Blue lures & Crowding-possible display & 1.11 & 0.8627 & 10.74 & 0.9768 \\
& Crowding-minimized display & 0.59 & 0.6429 & 6.29 & 0.8754 \\
\hline
\end{tabular}

The linear and log slopes are computed including the target only condition 


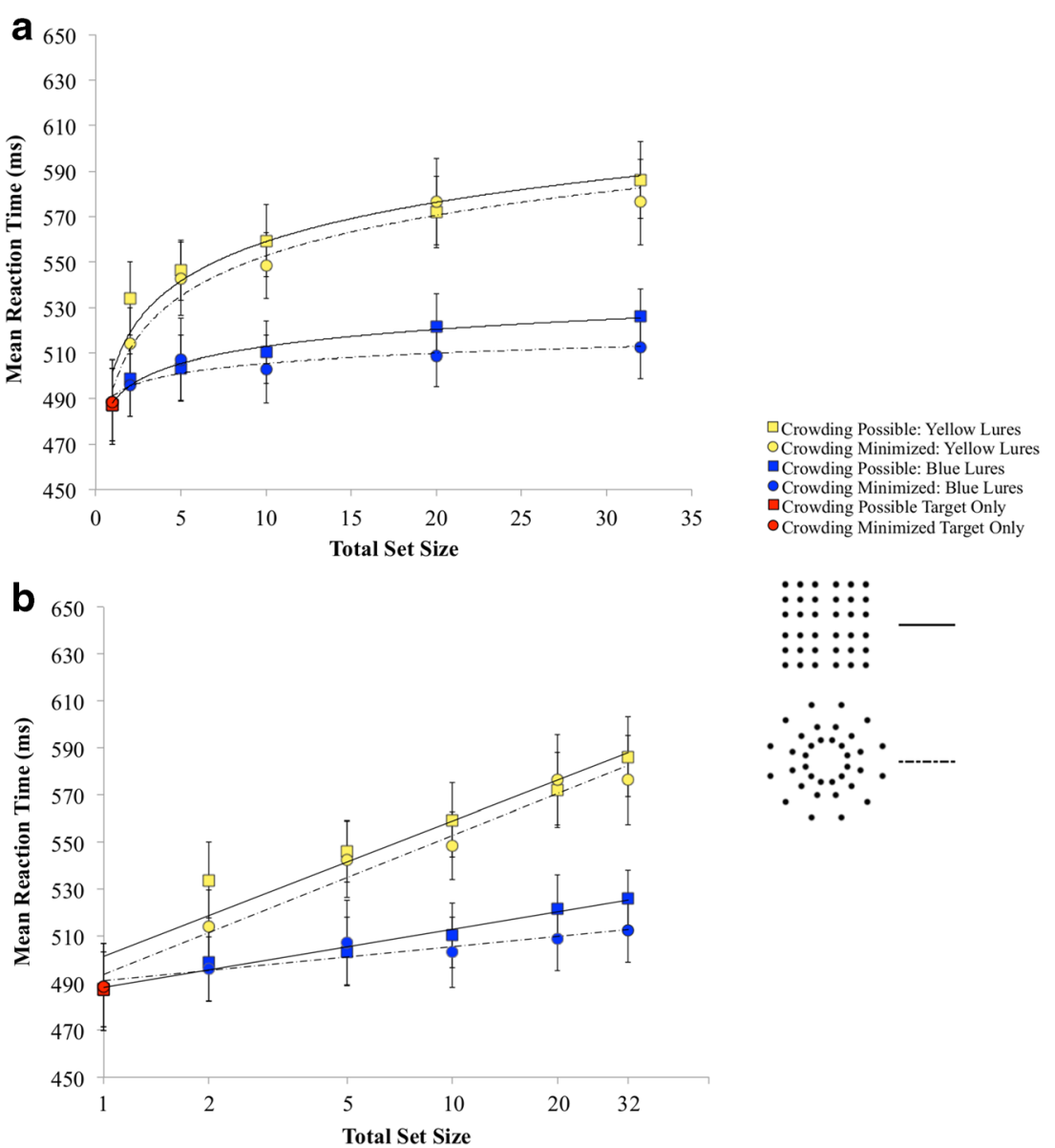

Fig. 4 a,b Group mean search performance (RT) as a function of interitem spacing, set size and lure-target similarity from Experiment 2. Error bars SEM. a Group mean search performance (RT) plotted on a linear scale for set size, showing the logarithmic relationship between the total number of items on the display and RT (ms). b Same data plotted on logarithmic scale for set size with the corresponding total set size conditions as labels than lures more similar to the target (yellow triangles) $\mathrm{F}(1,19)$ $=44.48, P<0.001$, Cohen's $\mathrm{d}=3.06$. The display type by lure type interaction was not significant $\mathrm{F}(1,19)=2.30, P=0.15$, Cohen's d $=0.70$, as in Experiment 1 .

Paired $t$ tests were conducted on the logarithmic slopes to evaluate the effect of display type on processing efficiency (Fig. 5). Display type failed to significantly alter search efficiency for either lure type. A paired $t$ test of the yellow triangle logarithmic slopes from the crowdingpossible $(25.0 \mathrm{~ms}$ per $\log$ unit) and crowdingminimized (25.6 ms per log unit) displays yielded no reliable difference in search efficiency between the two conditions, $\mathrm{t}(19)=0.165, P=0.871$, Cohen's $\mathrm{d}=0.05$. Similarly, a paired $t$ test of the blue circle logarithmic slopes for the crowding-possible ( $10.74 \mathrm{~ms}$ per log unit) and crowding-minimized (6.3 ms per log unit) displays was not significant, $\mathrm{t}(19)=1.105, P=0.283$, Cohen's $\mathrm{d}=$ 0.35 .

Finally, the effect of lure-target similarity on processing efficiency was evaluated for each display type. Paired $t$ tests were conducted on the logarithmic slopes produced by each lure item for both displays. For both display types, the logarithmic slope was larger for yellow triangle lures than for blue circle lures. On the crowding-minimized display, yellow triangles ( $25.7 \mathrm{~ms}$ per log unit) were process less efficiently than blue circles (6.3 ms per log unit), $\mathrm{t}(19)$ $=8.17, P<0.001$, Cohen's $d=2.58$. Similarly, on the crowding-possible display, yellow triangle lures $(25.0 \mathrm{~ms}$ per log unit) were processed less efficiently than blue circle lures (10.7 ms per log unit), t(19) $=3.99, P<0.001$, Cohen's $d=1.26$.

\section{Error rates}

As in the previous experiment, error rates were low (M: $1.31 \%$, SD: $0.74 \%$ ), and all but one subject had an error rates below 5\%. A repeated measures ANOVA was conducted on the error rates with display (crowding possible or minimized), lure (yellow triangles or blue circles), and set size $(2,5,10,20,32)$ as within-subject factors. Display 


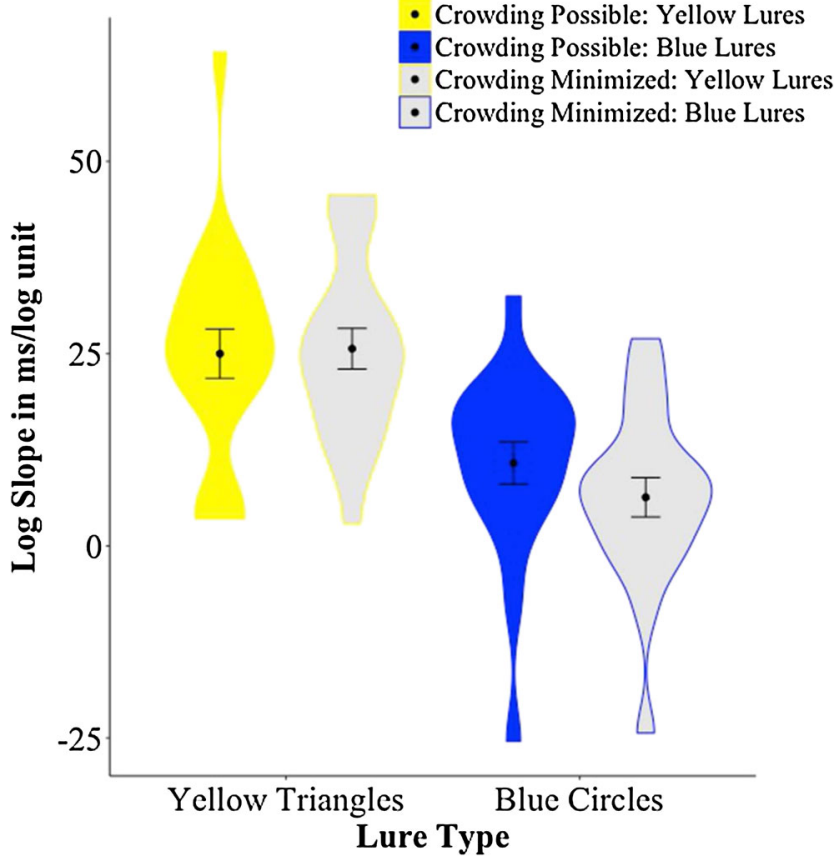

Fig. 5 Violin plots of the logarithmic slope coefficients from Experiment 2 shown for both display types grouped by lure type. The mean $\log$ slope is indicated by the dot on each plot. Error bars SEM

type $\mathrm{F}(1,19)=0.89, P=0.36$, Cohen's $\mathrm{D}=0.43$, and set size had no effect on accuracy $\mathrm{F}(2.836,53.887)=2.37, P=$ 0.084 , Cohen's $\mathrm{D}=0.71$, but lure type did $\mathrm{F}(1,19)=$ $12.70, P=0.002$, Cohen's $\mathrm{D}=1.64$. All of the interactions failed to reach significance $\mathrm{Fs}_{\mathrm{s}}<1.30$ and $P_{\mathrm{S}}>0.28$. Paired $t$ tests indicated the yellow triangle error rates were higher than the blue circle error rate on in both the crowdingpossible $\mathrm{t}(19)=2.86, P=0.01$, Cohen's $\mathrm{D}=0.9$ and crowding-minimized conditions $\mathrm{t}(19)=3.96, P=0.001$, Cohen's D $=1.23$. This pattern of results is not consistent with a speed-accuracy trade-off since yellow triangles had longer RTs and higher error rates.

\section{Eccentricity effects}

We performed the analogous exploratory analysis looking at the effect of eccentricity on target processing (Appendix 1: Fig. 10 and Fig. 11). Eccentricity had a significant effect on RT in both the crowding-possible display, $\mathrm{F}(2,38)=77.78, P$ $<0.001$, Cohen's $\mathrm{d}=0.85$, and the crowding-minimized display, $\mathrm{F}(2,38)=28.90, P<0.001$, Cohen's $\mathrm{d}=0.60$.

\section{Discussion}

The results from Experiment 2 provide converging evidence that the presence or absence of crowding on a search display does not qualitatively change the shape of the search function: once again, under conditions of minimized crowding, RT still increase logarithmically as a function of set size. Further, Experiment 2 demonstrates that, when lure-target similarity is sufficiently low, the contribution of crowding to RT performance in in fact minimal, with no measurable effects on search efficiency. Taken together, the results of Experiments 1 and 2 suggest the contribution of crowding to RT performance may vary as a function of lure-target similarity. These results are in line with previous studies on crowding reporting diminishing crowding effects when flanker-target similarity decreases (Ester et al., 2015; Kooi et al., 1994; Nazir, 1992; Põder, 2007; Scolari et al. 2007; Zahabi \& Arguin, 2014). In particular, Scolari et al. (2007) found a reduction in the critical spacing of items during a pop-out search task compared to a non-pop-out search task. That is, items in the pop-out search task had to be closer to the target to cause interference compared to the non-pop-out search task. Scolari and colleagues speculated this reduction in critical spacing might be due to pop-out facilitating the perceptual segregation of the target from distractors. Because we did not manipulate inter-item spacing, it is possible that larger crowding effects would have been found in this experiment had we used a smaller inter-item distance in our displays.

Finally, the effect of eccentricity on RT was replicated in Experiment 2, with significant effects of eccentricity in both the crowding-possible and crowding-minimized displays. The finding of eccentricity effects in the absence of measurable crowding effects suggests eccentricity can impact RT performance independently of crowding-related interference effects on perceptual processing. This finding is generally consistent with the known limitations of peripheral processing in vision (see Strasburger, Rentschler, \& Jüttner, 2011, for a review).

\section{Experiment 3}

The aim of Experiment 3 was to do a final test of the effects of crowding on parallel search performance, using within the same experiment both high (orange diamonds) and low (blue circles) lure-target similarity stimuli. Given the results of Experiments 1 and 2, we expected to find crowding to contribute more to RT performance in the orange diamond conditions compared to the blue circle conditions. We also expected search efficiency to be impacted by the possibility of crowding in the high, but not the low lure-target similarity conditions, consistent with the finding that crowding decreases as flanker-target similarity decreases (Andriessen \& Bouma, 1976; Ester et al., 2015; Kooi, et al., 1994; Nazir, 1992; Põder, 2007; Zahabi \& Arguin, 2014).

\section{Methods}

Methods were identical to Experiment 2 except where noted. 


\section{Subjects}

Data from 20 new subjects from the same population as Experiments 1 and 2, and who met the same performance criteria the previous experiments were analyzed. Two subjects were replaced according to our exclusion criteria: one subject was replaced for excessive RT, and another subject for high error rates.

\section{Apparatus and stimuli}

Lure objects in this experiment were the orange diamonds used in Experiment 1 and the blue circles triangles used in Experiment 2.

\section{Results}

\section{Search slopes}

As in Experiments 1 and 2, the search slopes were best approximated by logarithmic functions for both the crowdingpossible and crowding-minimized displays for both lure conditions (Table 3 and Fig. 6), as seen as higher $R$-squares for the logarithmic fit compared to the linear.

\section{Logarithmic search slopes}

Next, we evaluated the logarithmic search slopes (shown in Fig. 6a). For each logarithmic search slope, we fit participants' data with a logarithmic function that included the target-only datum. The effect of crowding and luretarget similarity on processing efficiency was evaluated with a $2 \times 2$ repeated measure ANOVA with display type and lure type as within-subject factors on the log slopes. Display type had a significant effect on log efficiency: crowding-minimized displays produced more efficient processing than crowding-possible displays $\mathrm{F}(1,19)=$ $10.64, P=0.0041$, Cohen's $\mathrm{d}=1.50$. Lure type also significantly impact log efficiency: lures less similar to the target (blue circles) produced more efficient process- ing than lures more similar to the target (orange diamonds) $\mathrm{F}(1,19)=194.14, P<0.001$, Cohen's $\mathrm{d}=6.39$. The display type by lure type interaction was not significant $\mathrm{F}(1,19)=2.40, P=0.14$, Cohen's $\mathrm{d}=0.71$. We had expected this interaction to be significant, but it was not. However, the estimated effect size is relatively large (0.7), so perhaps the absence of significance here merely implies inadequate power to detect that effect size with the current sample size.

Paired $t$ tests were conducted on the logarithmic slopes (computed separately for each subjects for each search condition; see Fig. 7) to best evaluate the effect of display type on processing efficiency. The paired $t$ tests revealed the orange diamond lures were processed more efficiently on crowdingminimized displays (31.1 ms per log unit) than when presented on the crowding-possible display (41.7 ms per log unit), $\mathrm{t}(19)=3.71 P=0.0015$, Cohen's $\mathrm{d}=1.17$. No reliable difference was found between the processing efficiency on blue circle in crowding-minimized (5.6 ms per log unit) compared to the crowding-possible displays (10.6 ms per log unit) displays, $\mathrm{t}(19)=1.59, P=0.128$, Cohen's $\mathrm{d}=0.5$, although the direction of the numerical difference was consistent with a modest effect of crowding on search efficiency (see above).

The effect of lure-target similarity on processing efficiency was confirmed by paired $t$ tests on the logarithmic slopes of each display condition between the two lure types. Across both display types, the logarithmic slopes were modulated by the lure-target similarity. For the crowding-possible display, orange diamond lures (41.7 ms per log unit) were processed less efficiently than blue circle lures (10.6 ms per log unit), $\mathrm{t}(19)=10.50, \mathrm{P}<$ 0.001, Cohen's $d=3.32$ ). Correspondingly in the crowdingminimized display, orange diamonds ( $31.1 \mathrm{~ms}$ per log unit) were processed less efficiently than blue circles $(5.6 \mathrm{~ms}$ per $\log$ unit), $\mathrm{t}(19)=10.30, \mathrm{p}<0.001$, Cohen's $\mathrm{d}=3.27$.

\section{Error rates}

Error rates were evaluated to rule out the possibility of a speedaccuracy trade-off. As in the previous two experiments, error

Table 3 Linear (in ms/item) and log slopes (in ms/log unit) results from Experiment 3 for each lure and display types are shown with the corresponding $R^{2}$

\begin{tabular}{|c|c|c|c|c|c|}
\hline & & Linear search slopes & $R^{2}$ & Log search slopes & $R^{2}$ \\
\hline \multirow[t]{2}{*}{ Orange lures } & Crowding-possible display & 4.51 & 0.9286 & 41.70 & 0.9651 \\
\hline & Crowding-minimized display & 3.20 & 0.8592 & 31.09 & 0.9847 \\
\hline \multirow[t]{2}{*}{ Blue lures } & Crowding-possible display & 1.11 & 0.8487 & 10.64 & 0.9442 \\
\hline & Crowding-minimized display & 0.59 & 0.7510 & 5.64 & 0.8374 \\
\hline
\end{tabular}

The linear and log slopes are shown computed including the target only condition 




Fig. 6 Search performance as a function of display type, set size and lure-target similarity on Experiment 3 using orange diamonds and blue circles. Error bars SEM. a Search performance plotted on a linear scale for set size, showing a logarithmic relationship between the total number

rates were general low (M:1.2\%, SD: $0.48 \%)$ with all but one subject having an error rate below 5\%. A repeated measures ANOVA with display type (crowding possible or minimized), lure type (orange diamonds or yellow triangles), and set size (2, $5,9,10,20,32)$ as within-subjects factors was conducted. There was no effect of display $\mathrm{F}(1,19)=0.12, \mathrm{p}=0.73$, Cohen's $\mathrm{D}=$ 0.16 , lure $\mathrm{F}(1,19)=0.46, P=0.51$, Cohen's $\mathrm{D}=0.31$, or set size $\mathrm{F}(2.569,48.808)=0.49, P=0.66$, Cohen's $\mathrm{D}=0.31$. The Display $\times$ Lure interaction was significant $\mathrm{F}(1,19)=4.81, P=$ 0.041 , Cohen's $D=1.01$. All other interactions were not significant Fs $<0.75$ and $P \mathrm{~S}>0.50$. Follow up paid $t$ tests between the display types and lure types were non significant $P_{\mathrm{S}}>0.059$. These results suggest there was no speed-accuracy trade-offs in this experiment.

\section{Eccentricity effects}

The effect of eccentricity was evaluated as previously done by testing three levels of eccentricity (Appendix 1: Fig. 12 and Fig. 13). The results indicated eccentricity had a significant effect on of items on the display and RT (ms). b Shows the same data plotted on logarithmic scale for set size with the corresponding total set size conditions as labels

RT in both the crowding-possible display, $\mathrm{F}(2,38)=38.36, P<$ 0.001 , Cohen's $\mathrm{d}=0.82$, and crowding-minimized display, $\mathrm{F}(2$, $38)=15.95, P<0.001$, Cohen's $d=0.45$. Thus, targets presented at a larger eccentricity required more processing than objects presented at a smaller eccentricity.

\section{Discussion}

The results of Experiment 3 are a final confirmation that the presence or absence of crowding in search displays does not fundamentally alter the search function: under conditions of efficient search with a fixed target, RTs increase logarithmically with set size, whether items crowd each other or not. The current results also replicate the findings of Experiment 1 : the presence of crowding in a display can reduce logarithmic search efficiency (i.e., increase the log slope), and this effect is particularly visible when lure-target similarity is relatively high (as between orange lures and the red target). The results suggest that when crowding is possible in a display, it 


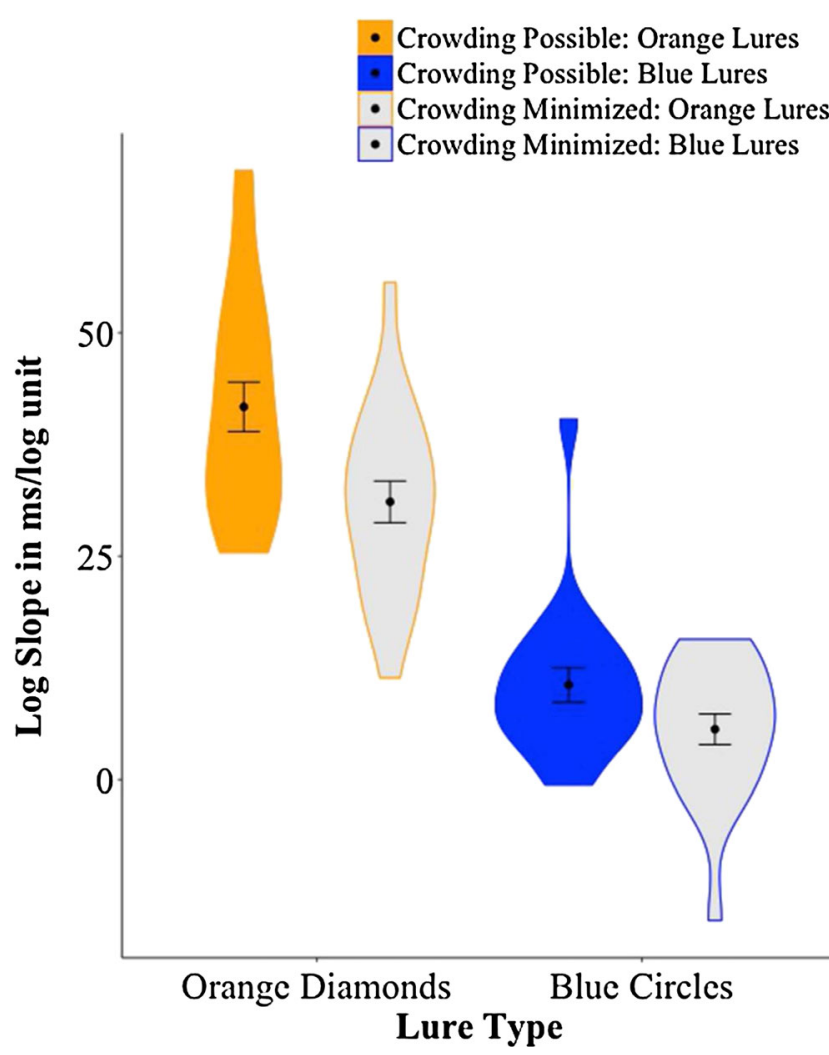

Fig. 7 Violin plots of the logarithmic slope coefficients from Experiment 3 are shown for both display types grouped by lure type. The mean log slope is indicated by the dot on each plot. Error bars SEM

may slow down the accumulation of perceptual evidence required to locate the target in the display.

\section{General discussion}

Buetti et al. (2016) documented that, in efficient search, when the target is fixed across trials, RTs increase logarithmically with set size. They proposed that this pattern of results reflected processing in a parallel, unlimited capacity and resolution limited architecture. However, recent work by Rosenholtz and colleagues (Chang \& Rosenholtz, 2016; Rosenholtz et al., 2012a,b) suggested a potential alternate account for this pattern of results. Rosenholtz et al. (2012b) proposed that peripheral vision is best understood as processing information through "pooling" regions, that is, regions of the world where perceptual features are averaged (or pooled) together (rather than analyzed independently). They proposed this averaged information is the meaningful visual representation that guides attention and determines efficiency in visual search. They also argued these pooling regions are the reason why crowding exists: within a pooling region, it is difficult to identify a target from its flanking elements because the features of target and flankers are all averaged together and represented as a summary statistic. In our displays, all lures are identical to one another. Therefore, according to Rosenholtz's framework, one would expect then that in displays where crowding is possible, the effect on RT of adding one additional item to the display will differ as a function of overall set size. In other words, the marginal cost of adding an element to the display is not constant but rather, it should decrease with set size. At small set sizes, every additional item added to the display is more likely to fall in an empty pooling region. Processing this additional element should carry some cost associated with having to compute and extract information from one additional pooling region that was not previously contributing to performance. However, when we add an item to a display that already contains many items, chances are it will fall inside a pooling region that already contains (and is pooling) identical items. Thus, this additional item would not alter the summary statistics of that pooling region (or only minimally so). In sum, the Rosenholtz framework could have a priori explained why RTs increase in a negatively accelerated fashion as a function of set size in efficient search, when all non-target stimuli are identical to each other. However, the data from these three experiments argues against this "pooling regions" account of logarithmic efficiency. Each time, when the possibility of crowding in our displays was minimized, RTs continued to have a logarithmic relationship to set size.

Crowding did impact search efficiency. Data from Experiments 1 and 3 does support the conclusion that when lure-target similarity is sufficiently high, search becomes less efficient when lures in the display crowd each other than when they do not. Following the architecture proposed by Buetti et al. (2016, see also Wang, Buetti \& Lleras, 2017), increases in log slopes can be interpreted as the visual system requiring more evidence to distinguish lures from the target at each display location. Thus, when lures are close to each other, crowding appears to either slow down the speed of evidence accumulation at those locations and/or heighten the amount of evidence required to confidently reject a lure as not being the target. As lure-target similarity increases, the comparison between the lure and the target template becomes harder to make, and this is even harder when the lure representations are crowded. That being said, the change in efficiency directly attributable to crowding was relatively modest: the change in logarithmic slope from crowding- 
possible to crowding-minimized conditions with orange diamond lures was about $\sim 10-15 \mathrm{~ms}$ per $\log$ unit in Experiments 1 and 3 (about $1.5 \mathrm{~ms} /$ item if slopes were computed in a linear scale).

More generally, our results seem consistent with previous findings on crowding regarding the effect of flanker-target similarity on target recognition: flanking items with higher similarity to the target tend to produce stronger crowding effects (Andriessen \& Bouma, 1976; Ester et al., 2014, 2015; Kooi et al., 1994; Nazir, 1992). Here, when lure-target similarity was high (orange lures conditions in Experiments 1 and 3 ), performance generally suffered (both in terms of overall RT and slopes) compared to conditions when lure-target similarity was smaller (yellow and blue lures).

We can also point out that the results from Experiments 1-3 are inconsistent with a texture segmentation account of the logarithmic search slope findings. A texture segmentation account would suggest displays that facilitate texture segmentation should lead to more efficient processing, as texture segmentation is a pre-attentive process (Julesz, 1981). The crowdingpossible display, where items were placed in a grid-array, should allow for better texture segmentation than the crowding-minimized displays because items were overall closer to each other, with a fixed separation, and thus, should have provided a stronger "texture" cue than the concentric displays. Yet, our results demonstrated that search functions were logarithmic both when texture segmentation was facilitated compared to when it was less likely (in concentric displays). Similarly, lure-grouping effects do not seem to be the cause of the logarithmic functions in efficient search, because the gridarrays likely produce stronger grouping effects than the crowding-minimized displays, again due to the fact that interitem spacing was smaller in those displays. This is not to say that lure-lure interactions do not play a role in efficient search. They most certainly do (Wang, Buetti \& Lleras, 2017). However, neither the presence nor the magnitude of such effects appears to be responsible for the logarithmic nature of the search functions.

Finally, our exploratory analysis revealed the presence of clear eccentricity effects across all three experiments. This suggests there is a limitation to how fast and accurate the human visual system is able to process visual information in parallel in the periphery. These results are consistent with previous findings from Carrasco and colleagues (Carrasco et al., 1995, 1998). Carrasco et al. $(1995,1998)$ attributed these eccentricity effects to physiological differences in the fovea and peripheral of the human eye, suggesting target processing has higher resolution at fovea compared to peripheral eccentricity. This is overall consistent with the known limitations in peripheral visual processing (Strasburger et al., 2011). Thus, the known spatial resolution limitations associated with peripheral vision are likely to be the resolution limitation proposed by Buetti et al. (2016) as being characteristic of stage one's ability to distinguish in parallel lures from targets and target-like stimuli.

\section{Limitations}

One limitation of this study is that we did not directly manipulate inter-item spacing or target crowding. This was because the goal of the study was not to study the impact of target crowding on visual search directly. Rather, our goal was to see whether logarithmic RT functions emerge because of pooling regions in peripheral vision that end up minimizing the impact on RT of additional lures as set size increases. Therefore, we cannot exclude the possibility that crowding could play a larger role in efficient visual search even when yellow and blue lures are used, if smaller inter-item spacing is used. That is, perhaps if we had placed lures closer to the target stimulus, or if we had consistently placed lures around the target (at all set sizes), maybe we would have measured a larger impact of crowding on search efficiency. As it stands, however, this limitation does not change much the basic conclusions of this article: that inter-item crowding/peripheral pooling does not appear to be responsible for the logarithmic nature of the search function in efficient search with fixed targets, even if crowding itself appears to modulate to a modest extent the efficiency of parallel search.

This study did not monitor eye movements nor were there any instructions given to the participants about what to do with their eyes. It is possible subjects moved their eyes or prepared to move their eyes during the search task. This behavior would have had an effect on crowding as evidence shows that para-saccade and saccadic planning can influence critical spacing (Harrison et al., 2013). This may limit the inferences we can make from these results in that if participant moved their eyes or prepared an eyemovement, display crowding would be directly effected by the preparation of that eye-movement (Harrison et al., 2013). The clear effect of eccentricity effects on RTs suggests we can be somewhat confident that performance was determined for the most part by the location where items initially appeared in the display relative to initial (central) fixation.

Acknowledgements The experiments were designed by A.M., A.L., and S.B. A.M. programmed the experiments and analyzed the data. A.M., A.L., and S.B. contributed to the manuscript. Data and experimental code for the experiments is available on the Open Science Framework osf.io/vrwmg. 
Appendix 1: Effect of eccentricity on search performance

Experiment 1: Orange diamond and yellow triangles as lure items

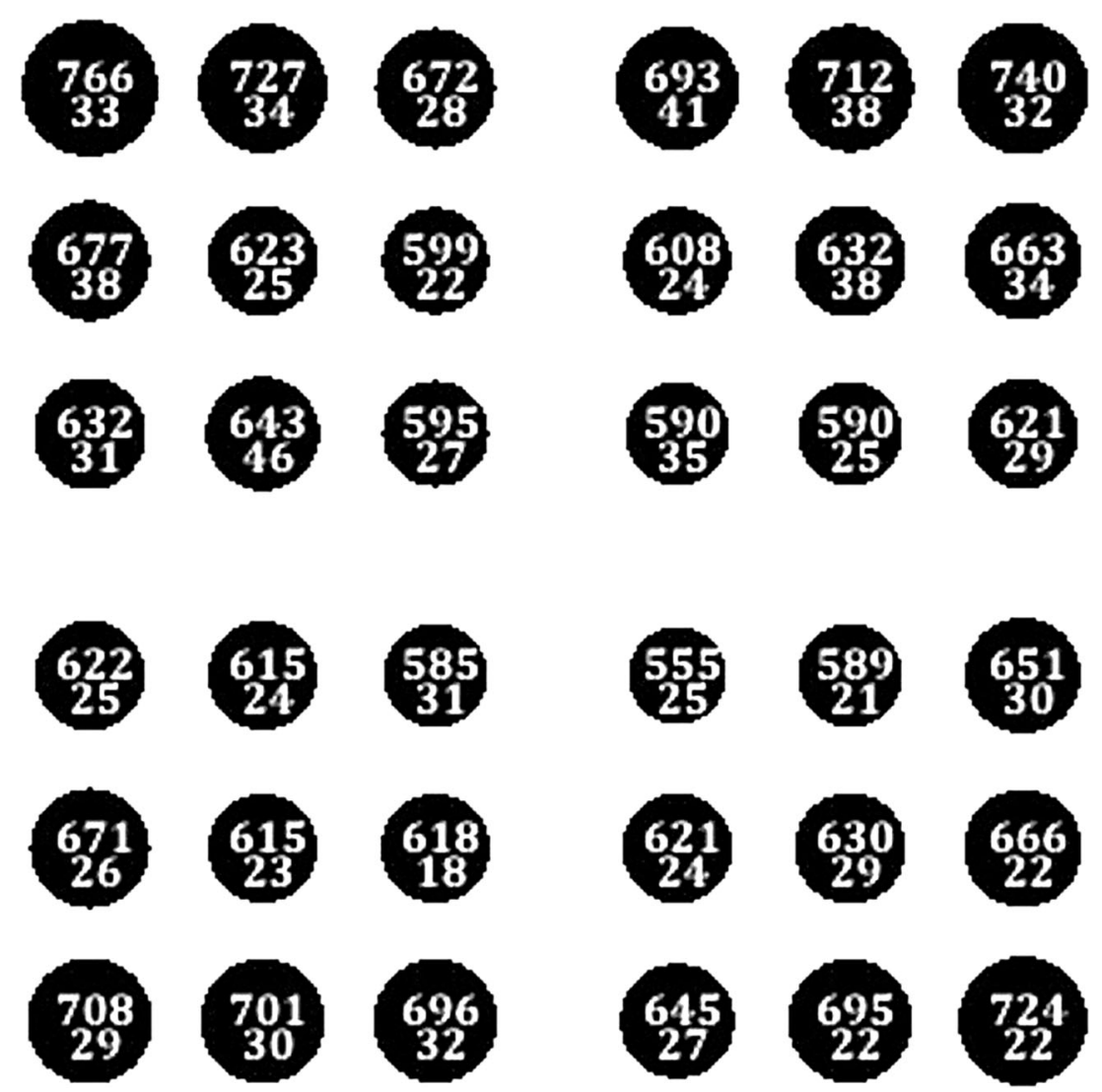

Fig. 8 Mean RT performance (in ms) on the crowding-possible display from Experiment 1 shown as a function of eccentricity collapsed across lure type and set size conditions. Each circle shows the mean RT in ms (top number) and SEM in ms (bottom number). The position of each

circle corresponds directly to the possible locations of the grid search array with the jitter removed. The diameter of each circle was made proportional to the mean RT. As can be seen, RTs increase as eccentricity of the target increases 


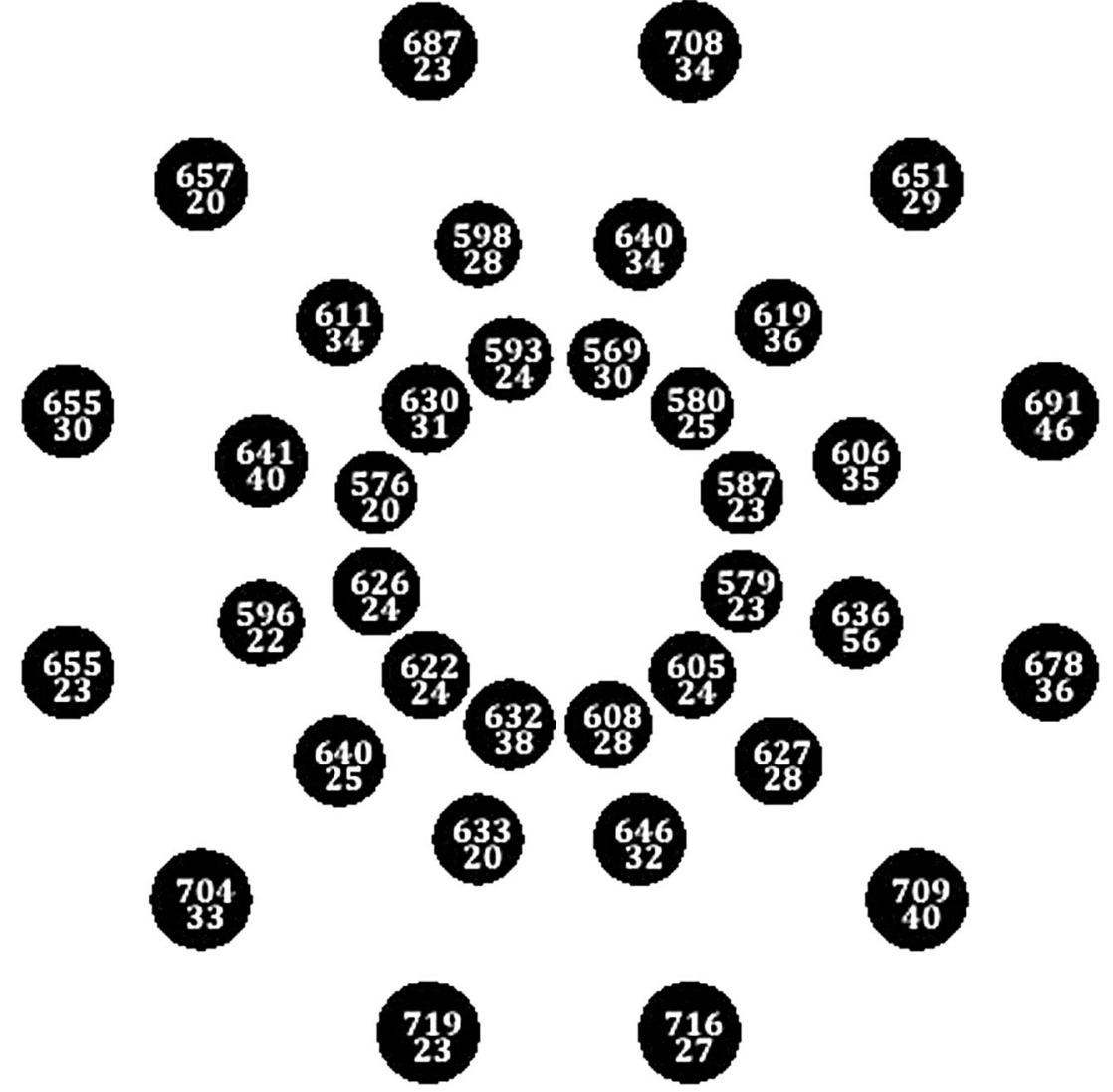

Fig. 9 Mean RT performance (in ms) on the crowding-minimized display from Experiment 1 shown as a function of eccentricity collapsed across lure type and set size condition. Each circle shows the mean RT in $\mathrm{ms}$ (top number) and SEM in ms (bottom number). The position of each circle corresponds directly to the possible locations of the concentric

search array with the jitter removed. The spacing between each object is proportional to the inter object distance in the displays. The diameter of each circle was made proportional to the mean RT. RTs increase as eccentricity of the target increases 
Experiment 2: Yellow triangles and blue circles as lure items.

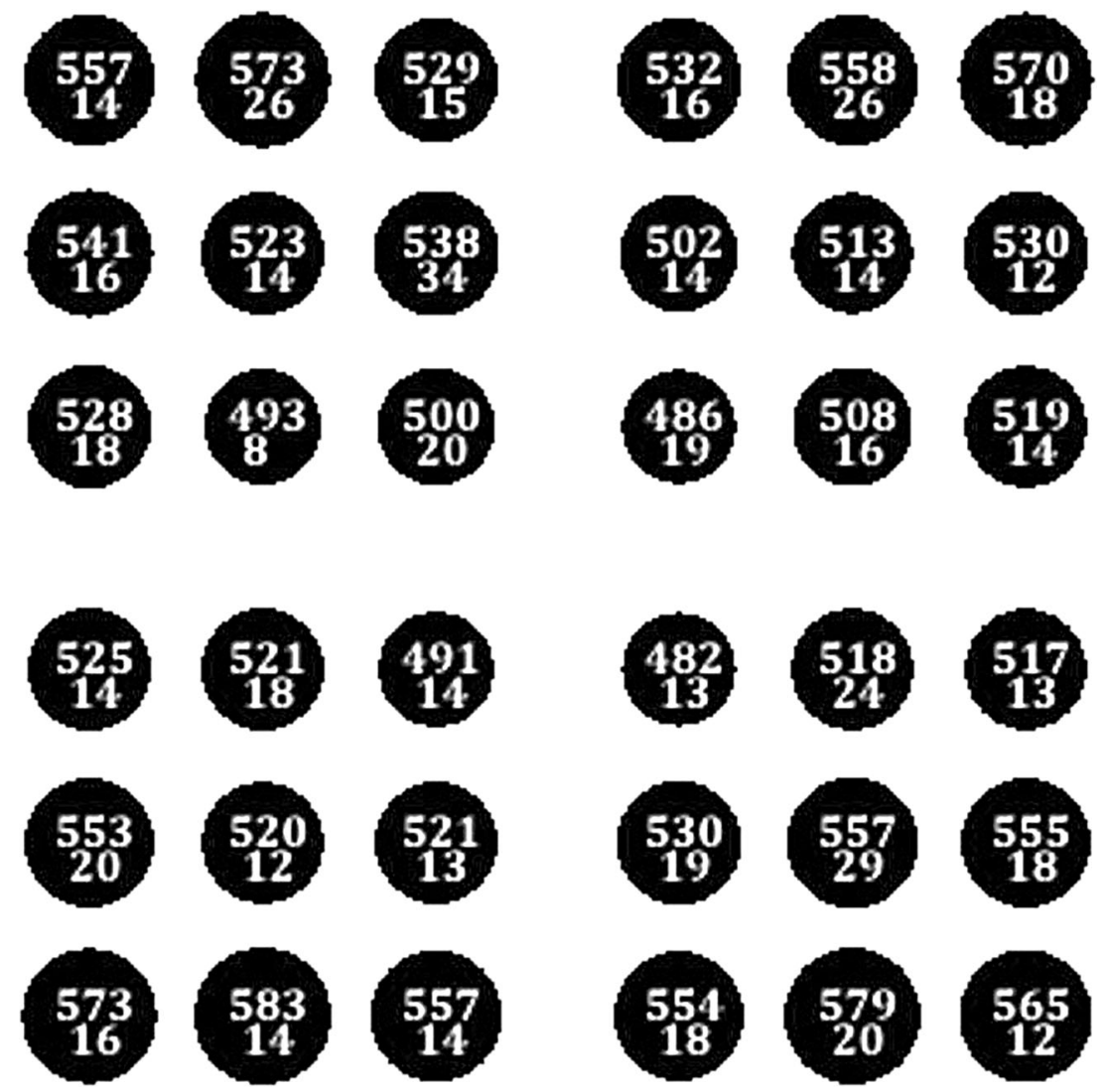

Fig. 10 Performance on the crowding-possible display in Experiment 2 as a function of eccentricity shown as mean RT (in ms) collapsed across lure type and set size conditions. Each circle shows the mean RT (top number) and SEM in ms (bottom number). The position of each circle

corresponds directly to the possible locations of the grid search array with the jitter removed. The diameter of each circle was made proportional to the mean RT. As can be seen, RTs increase as eccentricity of the target increases 
Experiment 3: Orange diamonds and blue circles

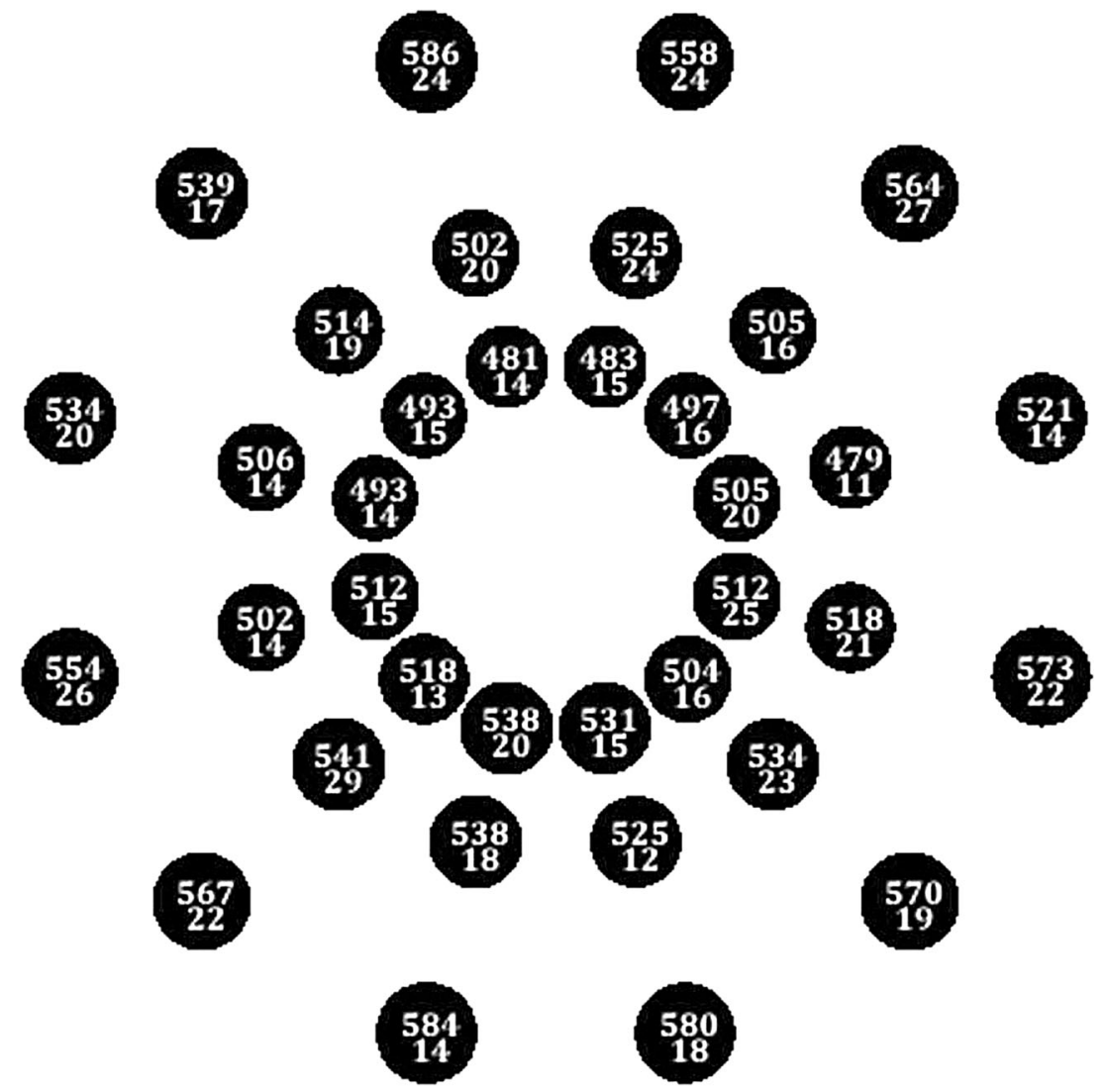

Fig. 11 As shown previously, performance on the crowding-minimized display in Experiment 2 as a function is shown as mean RT (in ms) collapsed across lure type and set size conditions. Each circle shows the mean RT in ms (top number) and SEM in ms (bottom number) for each location. The position of each circle corresponds directly to the possible

locations of the concentric search array with the jitter removed. The spacing between each object is proportional to the inter object distance in the displays. The diameter of each circle was made proportional to the mean RT. As can be seen, RTs increase as eccentricity of the target position increases 


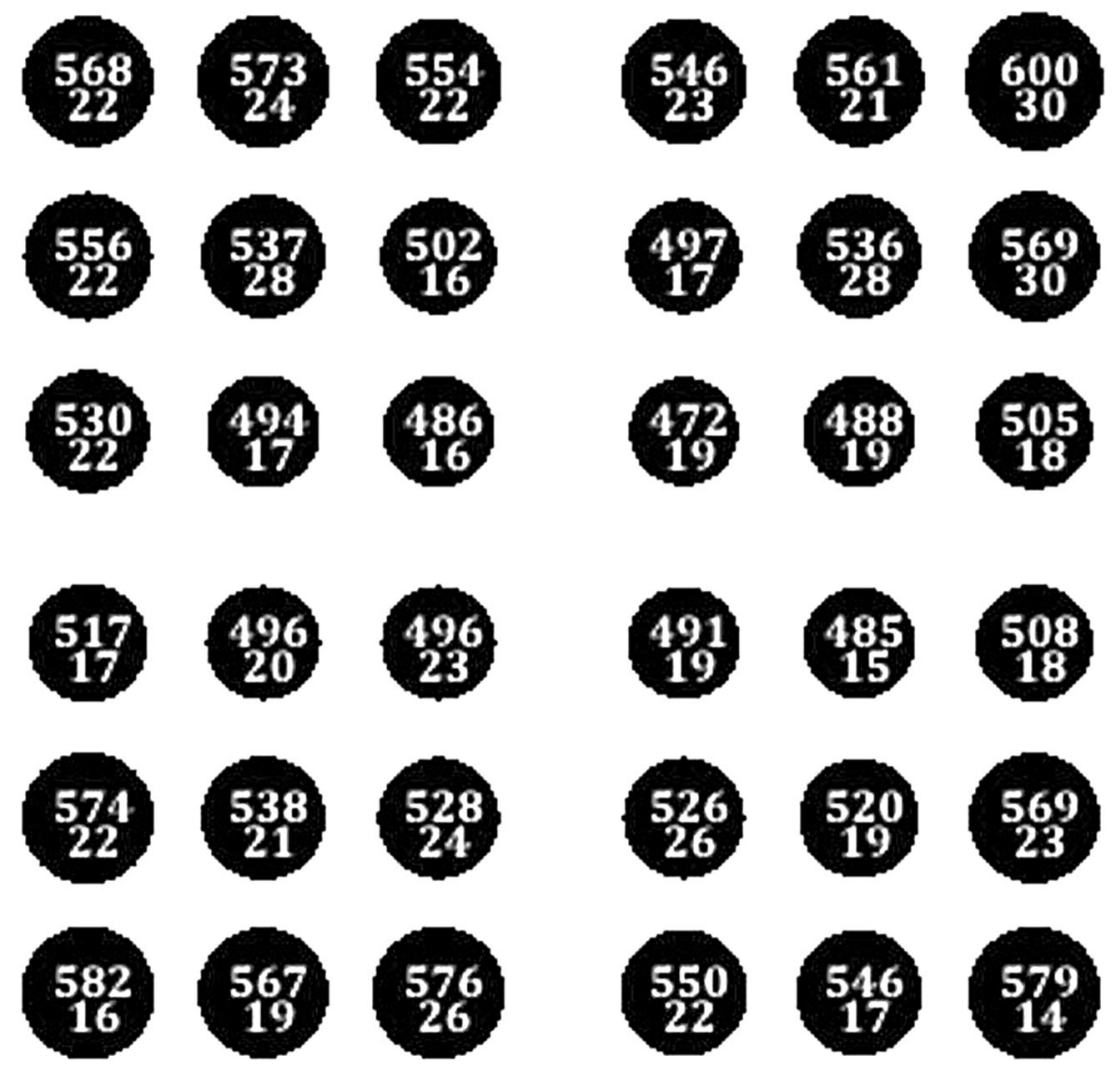

Fig. 12 As shown in previous figures, performance on the crowdingpossible display in Experiment 3 as a function of eccentricity is shown number). The position of each circle corresponds directly to the possible locations of the grid array with the jitter removed. The diameter of each circle was made proportional to the mean RT. As can be seen, RTs increase as eccentricity of the target increases 


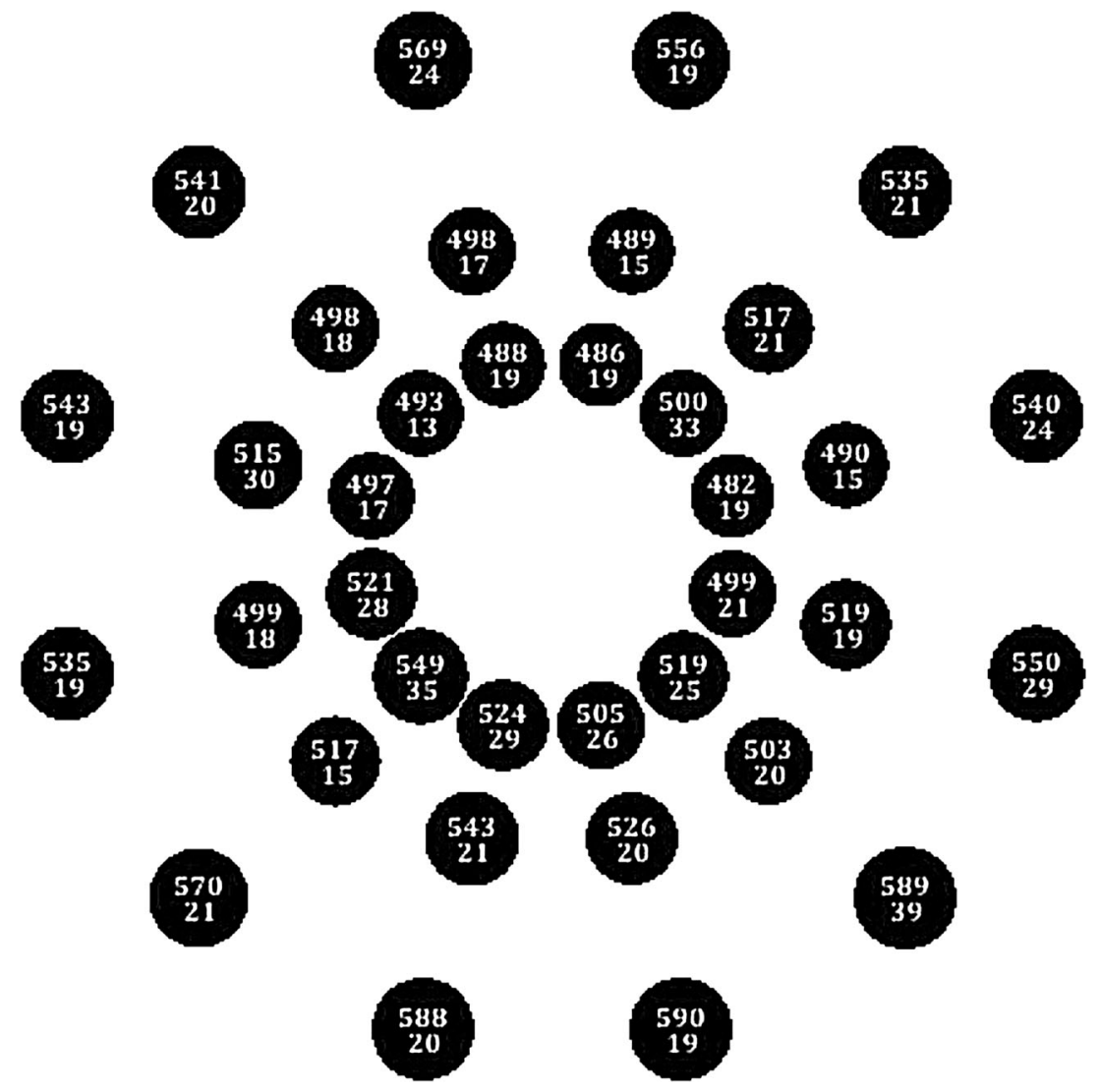

Fig. 13 As in the previous graphics, the performance on the crowdingminimized display in Experiment 3 as a function is shown as mean RT (in $\mathrm{ms}$ ). The results are collapsed across lure type and set size conditions. Each circle represents the mean RT in ms (top number) and SEM in ms (bottom number) for each location. The position of each circle

\section{References}

Andriessen, J. J., \& Bouma, H. (1976). Eccentric vision: Adverse interactions between line segments. Vision Research, 16(1), 71-78.

Balas, B., Nakano, L., \& Rosenholtz, R. (2009). A summary-statistic representation in peripheral vision explains visual crowding. Journal of Vision, 9(12), 13.1-18.

Brainard, D. H. (1997). The psychophysics toolbox. Spatial Vision, 10, 433-436.

Bouma, H. (1970). Interaction effects in parafoveal letter recognition. Nature, 226, 177-178.

Buetti, S., Cronin, D. A., Madison, A. M., Wang, Z., \& Lleras, A. (2016). Towards a better understanding of parallel visual processing in human vision: evidence for exhaustive analysis of visual information. Journal of Experimental Psychology: General. 145(6), 672-707.

Carrasco, M., Evert, D. L., Chang, I., \& Katz, S. M. (1995). The eccentricity effect: target eccentricity affects performance on conjunction searches. Perception \& Psychophysics, 57(8), 1241-1261.

Carrasco, M. \& Frieder, K. S. (1997). Cortical magnification neutralizes the eccentricity effect in visual search. Vision Research, 37(1), 63-82.

Carrasco, M., McLean, T. L., Katz, S. M., \& Frieder, K. S. (1998). Feature asymmetries in visual search: effects of display duration, target eccentricity, orientation and spatial frequency. Vision Research, 38(3), $347-374$.

corresponds directly to the possible locations of the concentric array with the jitter removed. The spacing between each object is proportional to the inter object distance in the displays. The diameter of each circle was made proportional to the mean RT. RTs increase as eccentricity of the target position increases

Carrasco, M., \& Yeshurun, Y. (1998). The contribution of covert attention to the set-size and eccentricity effects in visual search. Journal of Experimental Psychology. Human Perception and Performance, 24(2), 673-692.

Chang, H., Rosenholtz, R. (2016) Search performance is better predicted by tileability than presence of a unique basic feature. Journal of Vision, 16(10), 13.

Duncan, J., \& Humphreys, G. W. (1989). Visual search and stimulus similarity. Psychological Review, 96(3), 433-458.

Ester, E. F., Klee, D., \& Awh, E. (2014). Visual crowding cannot be wholly explained by feature pooling. Journal of Experimental Psychology: Human Perception and Performance, 40(3), 1022-1033.

Ester, E. F., Zilber, E., \& Serences, J. T. (2015). Substitution and pooling in visual crowding induced by similar and dissimilar distractors. Journal of Vision, 15(1):4, 1-12.

Freeman, J., \& Pelli, D. G. (2007). An escape from crowding. Journal of Vision, 7(2), 22.1-14.

Harrison, W. J., Mattingley, J. B., \& Remington, R. W. (2013). Eye movement targets are released from visual crowding. The Journal of Neuroscience, 33(7), 2927-2933.

Haslam, N., Porter, M., \& Rothschild, L. (2001). Visual search: efficiency continuum or distinct processes? Psychonomic Bulletin \& Review, 8(4), 742-746.

He, S., Cavanagh, P., \& Intriligator, J. (1996). Attentional resolution and the locus of visual awareness. Nature, 383(6598), 334-337. 
Hoffman, J. E. (1979). A two-stage model of visual search. Perception \& Psychophysics, 25(4), 319-327.

Hulleman, J., \& Olivers, C. (2015). The impending demise of the item in visual search. Behavioral and Brain Sciences, 17, 1-76.

Intriligator, J., \& Cavanagh, P. (2001). The spatial resolution of visual attention. Cognitive Psychology, 43(3), 171-216.

Itti, L., \& Koch, C. (2000). A saliency-based search mechanism for overt and covert shifts of visual attention. Vision Research, 40(10-12), $1489-1506$

Jonides, J. (1983). Further toward a model of the mind's eye's movement. Bulletin of the Psychnomic Society, 21(4), 247-250.

Julesz, B. (1981). Textons, the elements of texture perception, and their interactions. Nature, 290 (12), 91-97.

Kooi, F. L., Toet, A., Tripathy, S. P., \& Levi, D. M. (1994). The effect of similarity and duration on spatial interaction in peripheral vision. Spatial Vision, 8(2), 255-279.

Levi, D. M. (2008). Crowding-An essential bottleneck for object recognition: a mini-review. Vision Research, 48(5), 635-654.

Lleras, A., Buetti, S., \& Mordkoff, J. T. (2013). When do the effects of distractors provide a measure of distractibility? In B. H. Ross (Ed.), Psychology of Learning and Motivation (pp. 261-316). Amsterdam: Elsevier.

Nazir, T. A. (1992). Effects of lateral masking and spatial precueing on gap-resolution in central and peripheral vision. Vision Research, 32(4), 771-777.

Neider, M. B., \& Zelinsky, G. J. (2008). Exploring set size effects in scenes: identifying the objects of search. Visual Cognition, 16(1), $1-10$.

Neisser, U. (1964). Visual search. Scientific American, 210(6), 94-102.

Parkes, L., Lund, J., Angelucci, A, Solomon, J. A, \& Morgan, M. (2001). Compulsory averaging of crowded orientation signals in human vision. Nature Neuroscience, 4(7), 739-744.

Pelli, D. G. (1997). The VideoToolbox software for visual psychophysics: transforming numbers into movies. Spatial Vision, 10(4), 437-442.

Pelli, D. G. (2008). Crowding: a cortical constraint on object recognition. Current Opinion in Neurobiology, 18(4), 445-451.

Pelli, D. G., \& Tillman, K. A. (2008). The uncrowded window of object recognition. Nature Neuroscience, 11(10), 1129-1135.

Pelli, D. G., Palomares, M., \& Majaj, N. J. (2004). Crowding is unlike ordinary masking: distinguishing feature integration from detection. Journal of Vision, 4(12), 1136-1169.

Põder, E. (2007). Effect of colour pop-out on the recognition of letters in crowding conditions. Psychological Research, 71(6), 641-645.

Rosen, S., Chakravarthi, R., \& Pelli, D. G. (2014). The Bouma law of crowding, revised: critical spacing is equal across parts, not objects. Journal of Vision, 14(6), 1-15.

Rosenholtz, R., Huang, J., \& Ehinger, K. A. (2012a). Rethinking the role of top-down attention in vision: effects attributable to a lossy representation in peripheral vision. Frontiers in Psychology, 3, 1-15.
Rosenholtz, R., Huang, J., Raj, A., Balas, B. J., \& Ilie, L. (2012b). A summary statistic representation in peripheral vision explains visual search. Journal of Vision, 12(4), 1-17.

Santhi, N., \& Reeves, A. (2004). The roles of distractor noise and target certainty in search: a signal detection model. Vision Research, 44(12), 1235-1256.

Scolari, M., Kohnen, A., Barton, B., \& Awh, E. (2007). Spatial attention, preview, and pop-out: which factors influence critical spacing in crowded displays? Journal of Vision, 7(2), 1-23.

Strasburger, H., Rentschler, I., \& Jüttner, M. (2011). Peripheral vision and pattern recognition: a review. Journal of Vision, 11(5), 13.

Townsend, J. T., \& Ashby, F. G. (1983). The stochastic modeling of elementary psychological processes. Cambridge: Cambridge University Press.

Treisman, A., \& Gelade, G. (1980). A feature-integration theory of attention. Cognitive Psychology, 12(1), 97-136.

Treisman, A., \& Gormican, S. (1988). Feature analysis in early vision: evidence from search asymmetries. Psychological Review, 95(1), $15-48$.

Treisman, A., \& Souther, J. (1985). Search asymmetry: a diagnostic for preattentive processing of separable features. Journal of Experimental Psychology: General, 114(3), 285-310.

Toet, A., \& Levi, D. M. (1992). The two-dimensional shape of spatial interaction zones in the parafovea. Vision Research, 32(7), 1349-1357.

Tripathy, S. P., \& Cavanagh, P. (2002). The extent of crowding in peripheral vision does not scale with target size. Vision Research, 42(20), $2357-2369$.

Verghese, P. (2001). Visual search and attention: a signal detection theory approach. Neuron, 31, 523-535.

Wang, Z., Buetti, S., \& Lleras, A. (2017). Predicting search performance in heterogeneous visual search scenes with real-world objects. Collabra: Psychology, 3(1), 6.

Whitney, D., \& Levi, D. M. (2011). Visual crowding: a fundamental limit on conscious perception and object recognition. Trends in Cognitive Sciences, 15(4), 160-168.

Wolfe, J. M. (1994). Guided Search 2.0 A revised model of visual search. Psychonomic Bulletin \& Review, 1(2), 202-238.

Wolfe, J. M. (1998). Visual search. In H. Pashler (Ed.), Attention (pp. 141). London, UK.: University College London Press.

Wolfe, J. M., \& Gray, W. (2007). Guided search 4.0. Integrated models of cognitive systems, 99-119.

Wolfe, J. M., Võ, M. L.-H., Evans, K. K., \& Greene, M. R. (2011). Visual search in scenes involves selective and nonselective pathways. Trends in Cognitive Sciences, 15(2), 77-84.

Zahabi, S., \& Arguin, M. (2014). A crowdful of letters: disentangling the role of similarity, eccentricity and spatial frequencies in letter crowding. Vision Research, 97, 45-51.

Zelinsky, G. J. (2008). A theory of eye movements during target acquisition. Psychological Review, 115(4), 787-835. 